\title{
Effects of Aerobic Training Progression on Blood Pressure in Individuals With Hypertension: A Systematic Review With Meta-Analysis and Meta-Regression
}

\author{
Guilherme Tadeu de Barcelos' ( $\nabla$ guilherme_barcellos@hotmail.com ) \\ Universidade Federal de Santa Catarina \\ Isabel Heberle' \\ Universidade Federal de Santa Catarina \\ Juliana Cavestré Coneglian ${ }^{1}$ \\ Universidade Federal de Santa Catarina \\ Bruno Allan Vieira' \\ Universidade Federal de Santa Catarina \\ Rodrigo Sudatti Delevatti ${ }^{1}$ \\ Universidade Federal de Santa Catarina \\ Aline Mendes Gerage ${ }^{1}$ \\ Universidade Federal de Santa Catarina
}

\section{Research Article}

Keywords: Exercise, Cardiovascular diseases

Posted Date: February 8th, 2021

DOI: https://doi.org/10.21203/rs.3.rs-156120/v1

License: (c) (i) This work is licensed under a Creative Commons Attribution 4.0 International License. Read Full License

Version of Record: A version of this preprint was published at Frontiers in Sports and Active Living on February 17th, 2022. See the published version at https://doi.org/10.3389/fspor.2022.719063. 


\section{Abstract}

Objective: To analyze, through a systematic review with meta-analysis, the effects of aerobic training with and without progression on systolic blood pressure (SBP) and diastolic blood pressure (DBP) in hypertensive adults.

Method: The search for the studies was carried out in the PubMed, Cochrane Central, SPORTDiscus and LILACS databases. Clinical trials that analyzed the effect of aerobic training, lasting at least six weeks, on blood pressure in hypertensive individuals comparing with a control group without intervention were selected. The selection of studies and data extraction were carried out independently by two pairs of researchers.

Results: Of the 13028 studies found, 24 were selected and included in this review. There was a reduction in SBP after aerobic training with progression $(-10.67 \mathrm{mmHg} ; 95 \% \mathrm{Cl}-15.421,-5.926 ; \mathrm{p}<0.001)$ and without progression $(-10.17 \mathrm{mmHg} ; \mathrm{Cl}-12.213$, -8.120; $p$ < 0.001). DBP also decreased after aerobic training with progression $(-5.49 \mathrm{mmHg} ; 95 \% \mathrm{Cl}-8.663,-2.310 ; p<0.001)$ and without progression $(-6.51 \mathrm{mmHg} ; 95 \% \mathrm{Cl}-9.147,-3.868 ; \mathrm{p}<0.001)$.

Conclusion: Aerobic training promotes a reduction in the SBP and DBP levels of adults with hypertension, regardless of whether or not the training variables progression.

\section{Background}

Hypertension is a multifactorial chronic disease that affects more than one billion adults worldwide ${ }^{1}$, and is considered an important cardiovascular risk factor, since it is strongly associated with the occurrence of several other cardiovascular diseases $^{2}$ and mortality ${ }^{3}$. In addition to medication measures, changes in lifestyle are essential in the treatment of hypertension and include, among other aspects, the regular practice of physical exercises ${ }^{4}$.

In this regard, aerobic training of moderate intensity is the primary modality recommended in the management of hypertension (class of recommendation I and level of evidence A) which should be complemented by dynamic resistance training ${ }^{5-10}$. Previous review studies with meta-analysis showed significant mean reductions of 6.0 to $12.3 \mathrm{mmHg}$ in systolic blood pressure (SBP) and 3.4 to $6.1 \mathrm{mmHg}$ in diastolic blood pressure (DBP) in response to aerobic training in hypertensive individuals $^{11-13}$. However, despite including studies with different protocols, these review studies do not analyze the influence of the progression of training variables on blood pressure (BP). In addition, the main guidelines and organizations recommend the practice of aerobic exercises lasting 30 to 60 minutes a day or 150 minutes a week at moderate intensity, performed with a frequency of four to seven times a week ${ }^{4,5,9,10,14}$. Some guidelines also recommend this practice with different intensities and durations of the exercise ${ }^{10,14}$ and the gradual increase of these variables during a training program focused on the treatment of hypertension ${ }^{8}$, however the direction of these recommendations are only concerned with the dosage of these variables and not with their manipulation.

Some studies that compared the effects of different levels of these variables on aerobic training have shown reductions in $\mathrm{BP}$ regardless of the duration or intensity applied ${ }^{15-18}$, contradicting some indications that the reductions in BP occur in greater magnitude with higher training intensities ${ }^{19}$. Considering that higher intensities lead to significant benefits resulting from greater physiological adaptations ${ }^{19}$ not only in BP but in other aspects related to health ${ }^{17}$, it seems important, when planning a training program, to progress and reach higher intensities.

In addition, despite the fact that the practice of physical exercise with the recommended frequencies, durations and intensities causes beneficial effects, especially on cardiorespiratory fitness, the occurrence of a plateau in these effects interferes with the continuity of these benefits ${ }^{20}$, possibly associated with a greater state of training. The manipulation of training variables can be an important strategy for the continuity of health benefits; however, little is known about the effects of the progression of aerobic training variables in the health context, especially in the adaptations of BP in hypertensive 
adults. Thus, the objective of the present study was to analyze, through a systematic review with meta-analysis, the effects of aerobic training with and without progression in SBP and DBP of adults with hypertension.

\section{Method}

This study is characterized as a systematic review with meta-analysis and meta-regression of clinical trials. The study followed the items of PRISMA ${ }^{21}$ and was previously registered on the PROSPERO platform (CRD42020161767).

\subsection{Search for articles}

The PubMed, Cochrane Central, SPORTDiscus and LILACS databases were used to search for articles. The searches were carried out in December 2019 and there were no restrictions for the year of publication. The terms used for the search were "hypertension," "exercise" and "blood pressure," applied together. The Boolean operators "OR" and "AND" were used and the search was performed using the MeSH terms with their respective synonyms.

\subsection{Eligibility criteria}

Clinical trials published in Portuguese, Spanish and English, which included hypertensive adults ( $\geq 18$ years old), of both sexes, who participated in a supervised and structured aerobic exercise intervention for at least six weeks were considered eligible. There were no restrictions on the modality, intensity, session duration, volume and weekly frequency of aerobic training. Clinical trials should compare at least one group with aerobic exercise with a control group without exercise. Studies that contained co-interventions linked to training (e.g., nutritional counseling) were only included if such intervention was applied to both groups (exercise and control). To be eligible, studies should provide data on SBP and DBP at rest before and after the intervention, or the difference between the pre- and post-intervention means with their respective dispersion values. Only studies that provided clinical BP measurements were eligible. All studies that combined aerobic exercise with another type of physical exercise, that presented only the value of ambulatory BP or that included hypertensive individuals with severe heart disease were excluded.

\subsection{Study selection and data extraction}

In the first selection step, the titles and abstracts of the studies were read by four independent researchers (G.T.B, B.A.V, I.H and J.C.C) divided into pairs. Subsequently, the selected articles were compared between the researchers of each pair. In the next step, the texts were read in full by the peers and the studies were included or excluded according to the eligibility criteria previously established. Disagreements between the two researchers of each pair regarding the inclusion or exclusion of the studies were resolved by the fifth researcher (A.M.G).

Data extraction was performed independently by the same researchers, divided into pairs in the same way as in the previous steps. The extracted data were compared to avoid any error in the extraction process, with the disagreements resolved by the fifth researcher. For all studies, the extraction of data related to the characteristics of the sample included: sample size; sex; average age; body mass index (BMI); training status; presence of comorbidities; time of diagnosis of hypertension; nutritional co-intervention; and adverse events arising from the intervention. For the information related to the intervention, the following data were considered: time of intervention; modality; method; session duration; weekly frequency; intensity; adherence to training; and withdrawals. In addition, the number of progressions for intensity, frequency and/or duration of the sessions was extracted. Studies that clearly reported some kind of progression in the frequency, duration and/ or intensity of the session were classified as aerobic training with progression, and those that did not clearly report or did not progress in these variables were classified as aerobic training without progression. Regarding the study outcomes, the information extracted was: SBP and DBP, with mean and measure of dispersion, for the exercise and control groups, at pre- and post-intervenvion.

\subsection{Analysis of risk of bias}

The assessment of risk of bias was carried out independently by the same researchers, divided into pairs and the fifth reviewer was consulted to resolve the disagreements. The risk of bias was assessed according to the Cochrane Handbook 
$(2019)^{22}$, considering the following criteria: generation of random sequence; concealment of allocation; concealment of the assessment of outcomes; conducting analysis by intention to treat; and description of withdrawals and exclusions. The risk of bias was classified as: high risk - when methodological criteria, such as the proper generation of random sequences, were not reported or were not performed; low risk - when the methodological criteria were properly carried out; unclear risk - when there was no adequate description of the criteria, it was not possible to evaluate it as high or low risk.

\subsection{Data analysis}

The combined effect estimates were calculated using the difference between the baseline values and the end of the intervention, with their respective standard deviation values and number of participants analyzed. Studies that presented other measures of dispersion had the values converted to standard deviation. The results of the analyses are presented as mean difference with a $95 \%$ confidence interval, and the calculations were performed using the random effects model. The statistical heterogeneity of the effects was assessed using the $\mathrm{I}^{2}$ inconsistency test, considering values above $50 \%$ as high heterogeneity ${ }^{22}$.

Subgroup analyses were performed considering training progression (without progression, with progression, progression in intensity, progression in duration and progression in intensity and duration), sex, presence of comorbidities, use of medications, intervention period, training method (continuous or interval), modalities (walking / running, cycle ergometer, different modalities) and the training environment (terrestrial or aquatic). The meta-regression analysis was performed to investigate the influence of possible confounding factors on the responses of SBP and DBP, namely: mean age (years); BMI $\left(\mathrm{kg} / \mathrm{m}^{2}\right)$; number of users of antihypertensive drugs; SBP baseline; weekly frequency (number of sessions per week); weekly duration (minutes); and intervention period (weeks).

To represent the results, a forest plot was generated, with the average difference and $95 \%$ confidence interval. Statistical significance was considered to be $p<0.05$. All analyses were performed using the OpenMeta Analyst Software, version 10.10 .

\section{Results}

\subsection{Study selection}

Initially, 13028 studies were found by searching the databases. After removing duplicates, 10900 studies were selected to read titles and abstracts. At the end of the first stage, 173 studies were selected for full reading, with 149 being excluded. Thus, 24 studies were included in the final analysis, among which 12 studies were classified as progressive aerobic training ${ }^{23-34}$ and 12 studies were classified as nonprogressive aerobic training ${ }^{35-46}$. In addition, 4 studies were analyzed twice for presenting two groups of aerobic training $28,31,35$ or for performing analyses at two different moments ${ }^{27}$ (Fig. 1)

\subsection{Characteristics of the studies}

Considering all studies, 1207 participants were analyzed, of which 716 were involved in aerobic training and 491 were part of the control group. Most of the studies included adults of both sexes (41.7\%), six studies analyzed only male participants (25.0\%), another six analyzed only female participants (25.0\%) and two studies did not report this information (8.3\%). Regarding the training status, 15 studies included untrained or sedentary participants $(62.5 \%)$ and nine studies did not report this information (37.5\%). The average age of the participants varied between 38.1 and 73.5 years and the BMI showed values between 23.3 and $34.4 \mathrm{~kg} / \mathrm{m}^{2}$. The general information on the characteristics of the participants is shown in Table 1 . 


\begin{tabular}{|c|c|c|c|c|c|c|c|c|}
\hline Study & $\begin{array}{l}\text { Sample } \\
\text { size } \\
\text { (\% } \\
\text { women) }\end{array}$ & $\begin{array}{l}\text { Average } \\
\text { age } \\
\text { (years) }\end{array}$ & $\begin{array}{l}\text { BMI } \\
\left(\mathrm{kg} / \mathrm{m}^{2}\right)\end{array}$ & $\begin{array}{l}\text { Duration } \\
\text { of the } \\
\text { disease } \\
\text { (years) }\end{array}$ & $\begin{array}{l}\text { Trainability } \\
\text { status }\end{array}$ & Comorbidities & $\begin{array}{l}\text { Co- } \\
\text { nutritional } \\
\text { intervention }\end{array}$ & $\begin{array}{l}\text { Adverse } \\
\text { events }\end{array}$ \\
\hline \multicolumn{9}{|c|}{ Progressive aerobic training } \\
\hline \multirow{2}{*}{$\begin{array}{l}\text { Abdelaal } \\
\text { and } \\
\text { Mohamad, } \\
2014^{23}\end{array}$} & $\begin{array}{l}\text { E: } 20 \\
(60 \%)\end{array}$ & \multirow[t]{2}{*}{52.5} & $\begin{array}{l}\mathrm{E}: 34.6 \pm \\
1.1\end{array}$ & \multirow[t]{2}{*}{ NR } & \multirow[t]{2}{*}{ Sedentary } & \multirow[t]{2}{*}{$\begin{array}{l}\text { Obesity and } \\
\text { DM2 }\end{array}$} & \multirow[t]{2}{*}{ No } & \multirow[t]{2}{*}{$\begin{array}{l}\text { No adverse } \\
\text { events } \\
\text { recorded }\end{array}$} \\
\hline & $\begin{array}{l}\text { C: } 19 \\
(53 \%)\end{array}$ & & $\begin{array}{l}\text { C: } 34.1 \pm \\
1.2\end{array}$ & & & & & \\
\hline \multirow{2}{*}{$\begin{array}{l}\text { Baghaiee } \\
\text { et al., } \\
2018^{24}\end{array}$} & $\begin{array}{l}\text { E: } 20 \\
(0 \%)\end{array}$ & \multirow[t]{2}{*}{38.1} & $\begin{array}{l}\mathrm{E}: 26.8 \pm \\
2.1\end{array}$ & \multirow[t]{2}{*}{ NR } & \multirow[t]{2}{*}{ Untrained } & \multirow[t]{2}{*}{ NR } & \multirow[t]{2}{*}{ No } & \multirow[t]{2}{*}{ NR } \\
\hline & $\begin{array}{l}\text { C: } 20 \\
(0 \%)\end{array}$ & & $\begin{array}{l}\text { C: } 27.2 \\
\pm 1.3\end{array}$ & & & & & \\
\hline \multirow{2}{*}{$\begin{array}{l}\text { Farahani } \\
\text { et al., } \\
2010^{25}\end{array}$} & $\begin{array}{l}\text { E: } 12 \\
(0 \%)\end{array}$ & \multirow[t]{2}{*}{47.7} & $\begin{array}{l}\text { E: } 27.4 \pm \\
4.3\end{array}$ & \multirow[t]{2}{*}{ NR } & \multirow[t]{2}{*}{ NR } & \multirow[t]{2}{*}{ No } & \multirow[t]{2}{*}{ No } & \multirow[t]{2}{*}{ NR } \\
\hline & $\begin{array}{l}\text { C: } 28 \\
(0 \%)\end{array}$ & & $\begin{array}{l}\text { C: } 28.1 \pm \\
3.5\end{array}$ & & & & & \\
\hline \multirow{2}{*}{$\begin{array}{l}\text { Hagberg } \\
\text { et al., } \\
1989^{26}\end{array}$} & $\begin{array}{l}\text { E: } 10 \\
\text { (NR) }\end{array}$ & \multirow[t]{2}{*}{64.4} & NR & \multirow[t]{2}{*}{ NR } & \multirow[t]{2}{*}{ NR } & \multirow[t]{2}{*}{ NR } & \multirow[t]{2}{*}{ No } & \multirow[t]{2}{*}{ NR } \\
\hline & $\begin{array}{l}\text { C: NR } \\
\text { (NR) }\end{array}$ & & & & & & & \\
\hline \multirow{4}{*}{$\begin{array}{l}\text { Kokkinos } \\
\text { et al., } \\
1995^{27}\end{array}$} & $\begin{array}{l}E_{16}: 18 \\
(0 \%)\end{array}$ & \multirow[t]{4}{*}{57.5} & $\begin{array}{l}E_{16}: 31.0 \\
\pm 5.5\end{array}$ & \multirow[t]{4}{*}{ NR } & \multirow[t]{4}{*}{ Sedentary } & \multirow[t]{4}{*}{ No } & \multirow[t]{4}{*}{ No } & NR \\
\hline & $\begin{array}{l}C_{16}: 14 \\
(0 \%)\end{array}$ & & $\begin{array}{l}\mathrm{C}_{16}: \\
31.0 \pm \\
5.5\end{array}$ & & & & & \\
\hline & $\begin{array}{l}E_{32}: 20 \\
(0 \%)\end{array}$ & & $\begin{array}{l}E_{32}: 31.0 \\
\pm 4.3\end{array}$ & & & & & \\
\hline & $\begin{array}{l}\mathrm{C}_{32}: 18 \\
(0 \%)\end{array}$ & & $\begin{array}{l}C_{32}: \\
31.0 \pm \\
4.3\end{array}$ & & & & & \\
\hline $\begin{array}{l}\text { Lamina et } \\
\text { al., } 2010^{28}\end{array}$ & $\begin{array}{l}E_{\text {CONT: }} \\
112 \\
(0 \%)\end{array}$ & 58.4 & $\begin{array}{l}\mathrm{E}_{\mathrm{CONT}}: \\
25.0 \pm \\
3.9\end{array}$ & $>1.0$ & Sedentary & No & No & $\begin{array}{l}\text { Unfavorable } \\
\text { responses } \\
\text { to training }\end{array}$ \\
\hline & $\begin{array}{l}E_{I N T}: \\
140 \\
(0 \%)\end{array}$ & & $\begin{array}{l}\mathrm{E}_{\mathrm{INT}}: \\
22.5 \pm \\
2.9\end{array}$ & & & & & \\
\hline & $\begin{array}{l}\text { C: } 105 \\
(0 \%)\end{array}$ & & $\begin{array}{l}\text { C: } 24.2 \\
\pm 4.9\end{array}$ & & & & & \\
\hline $\begin{array}{l}\text { Latosik et } \\
\text { al., } 2014^{29}\end{array}$ & $\begin{array}{l}\text { E: } 15 \\
(100 \%)\end{array}$ & NR & $\begin{array}{l}\text { E: } 28.2 \pm \\
6.3\end{array}$ & NR & NR & No & Yes & NR \\
\hline & $\begin{array}{l}\text { C: } 10 \\
(100 \%)\end{array}$ & & $\begin{array}{l}\text { C: } 28.2 \pm \\
5.5\end{array}$ & & & & & \\
\hline $\begin{array}{l}\text { Meirelles } \\
\text { et al., } \\
2009^{30}\end{array}$ & $\begin{array}{l}\text { E: } 13 \\
(61.5 \%)\end{array}$ & 49.5 & $\mathrm{E}: 30 \pm 1$ & NR & Sedentary & NR & No & NR \\
\hline
\end{tabular}




\begin{tabular}{|c|c|c|c|c|c|c|c|c|}
\hline & $\begin{array}{l}\text { C: } 6 \\
(66.6 \%)\end{array}$ & & $\begin{array}{l}\text { C: } 32 \pm \\
2\end{array}$ & & & & & \\
\hline $\begin{array}{l}\text { Soltani et } \\
\text { al., } 2019^{31}\end{array}$ & $\begin{array}{l}E_{\text {SHORT: }}: \\
10(0 \%) \\
E_{\text {LONG: }}: \\
10(0 \%) \\
\text { C: } 10 \\
(0 \%)\end{array}$ & 47.9 & $\begin{array}{l}\text { E }_{\text {SHORT: }} \\
30.0 \pm \\
2.3 \\
\text { E }_{\text {LONG: }}: \\
27.3 \pm \\
2.4 \\
\text { C: } 29.3 \\
\pm 2.3\end{array}$ & NR & Untrained & NR & No & NR \\
\hline $\begin{array}{l}\text { Tanaka et } \\
\text { al., } 1997^{32}\end{array}$ & $\begin{array}{l}\text { E: } 12 \\
(41.7 \%) \\
\text { C: } 6 \\
(50 \%)\end{array}$ & 48.0 & NR & NR & Untrained & Obesity & No & NR \\
\hline $\begin{array}{l}\text { Turner et } \\
\text { al., } 2000^{33}\end{array}$ & $\begin{array}{l}\text { E: } 11 \\
(18.2 \%) \\
\text { C: } 7 \\
(28.6 \%)\end{array}$ & 66.9 & $\begin{array}{l}\text { E: } 30.2 \pm \\
1.8 \\
\text { C: } 29.6 \pm \\
1.4\end{array}$ & $\begin{array}{l}\text { E: } 4.5 \pm \\
\text { 2. } 7 \\
\text { C: } 3.0 \pm \\
\text { 1. } 0\end{array}$ & Sedentary & No & No & $\mathrm{NR}$ \\
\hline $\begin{array}{l}\text { Wong et } \\
\text { al., } 2018^{34}\end{array}$ & $\begin{array}{l}\text { E: } 52 \\
(100 \%) \\
\text { C: } 48 \\
(100 \%)\end{array}$ & 73.5 & $\begin{array}{l}\text { E: } 26.0 \pm \\
2.8 \\
\text { C: } 26.9 \\
\pm 2.9\end{array}$ & NR & Sedentary & NR & No & $\begin{array}{l}\text { No adverse } \\
\text { events } \\
\text { recorded }\end{array}$ \\
\hline Non-progre & ive aerob & training & & & & & & \\
\hline $\begin{array}{l}\text { Arca et al., } \\
2014^{35}\end{array}$ & $\begin{array}{l}\text { E }_{\text {LAND: }}: \\
19 \\
(100 \%) \\
\text { E }_{\text {WATER }} \\
19 \\
(100 \%) \\
\text { C: } 14 \\
(100 \%)\end{array}$ & 64.0 & $\begin{array}{l}\text { E }_{\text {LAND }}: \\
28.3 \pm \\
4.2 \\
\text { E }_{\text {WATER }}: \\
27.0 \pm \\
5.1 \\
\text { C: } 30.9 \\
\pm 4.8\end{array}$ & NR & Untrained & DM2 $(n=5)$ & No & NR \\
\hline $\begin{array}{l}\text { He et al., } \\
2018^{36}\end{array}$ & $\begin{array}{l}\text { E: } 20 \\
(100 \%) \\
\text { C: } 22 \\
(100 \%)\end{array}$ & 57.5 & $\begin{array}{l}\text { E: } 27.4 \pm \\
2.1 \\
\text { C: } 27.7 \\
\pm 2.6\end{array}$ & NR & Untrained & No & No & NR \\
\hline $\begin{array}{l}\text { Hong et } \\
\text { al., } 2018^{37}\end{array}$ & $\begin{array}{l}\text { E: } 7 \\
(0 \%) \\
\text { C: } 7 \\
(0 \%)\end{array}$ & 51.3 & NR & NR & NR & No & No & NR \\
\hline $\begin{array}{l}\text { Khalid et } \\
\text { al.,2013 }\end{array}$ & $\begin{array}{l}\text { E: } 12 \\
(100 \%) \\
\text { C: } 13 \\
(100 \%)\end{array}$ & 52.8 & $\begin{array}{l}\text { E: } 34.9 \pm \\
3.5 \\
\text { C: } 33.8 \\
\pm 4.1\end{array}$ & NR & Sedentary & Obesity & No & NR \\
\hline
\end{tabular}




\begin{tabular}{|c|c|c|c|c|c|c|c|c|}
\hline \multirow[t]{4}{*}{$\begin{array}{l}\text { Izadi et al., } \\
2017^{39}\end{array}$} & $\begin{array}{l}\mathrm{E}: 15 \\
(46.7 \%)\end{array}$ & 61.6 & $\begin{array}{l}\text { E: } 25.2 \pm \\
0.6 \\
\text { (Men) }\end{array}$ & NR & Untrained & No & No & NR \\
\hline & $\begin{array}{l}\text { C: } 15 \\
(40 \%)\end{array}$ & & $\begin{array}{l}{ }^{25.7 \pm} \\
0.7 \\
\text { (Women) }\end{array}$ & & & & & \\
\hline & & & $\begin{array}{l}\text { C: } 25.2 \pm \\
0.8 \\
\text { (Men) }\end{array}$ & & & & & \\
\hline & & & $\begin{array}{l}25.3 \pm \\
1.2 \\
\text { (Women) }\end{array}$ & & & & & \\
\hline \multirow[t]{2}{*}{$\begin{array}{l}\text { Koga et } \\
\text { al., } 1992^{40}\end{array}$} & $\begin{array}{l}\text { E: } 10 \\
(100 \%)\end{array}$ & 49. 0 & NR & NR & $N R$ & No & No & NR \\
\hline & $\begin{array}{l}\text { C: } 5 \\
(100 \%)\end{array}$ & & & & & & & \\
\hline \multirow[t]{2}{*}{$\begin{array}{l}\text { Maruf et } \\
\text { al., } 2014^{41}\end{array}$} & $\begin{array}{l}\text { E: } 45 \\
\text { (NR) }\end{array}$ & 52. 0 & $\begin{array}{l}\text { E: } 27.5 \pm \\
5\end{array}$ & NR & NR & No & No & $\begin{array}{l}\text { Knee joint } \\
\text { pain }(n=2)\end{array}$ \\
\hline & $\begin{array}{l}\text { C: } 43 \\
\text { (NR) }\end{array}$ & & $\begin{array}{l}\text { C: } 25.4 \pm \\
4.7\end{array}$ & & & & & \\
\hline \multirow[t]{2}{*}{$\begin{array}{l}\text { Miura et } \\
\text { al., } 1994^{42}\end{array}$} & $\begin{array}{l}\text { E: } 17 \\
(88.2 \%)\end{array}$ & 49. 0 & NR & NR & NR & No & No & NR \\
\hline & $\begin{array}{l}\text { C: } 10 \\
(90 \%)\end{array}$ & & & & & & & \\
\hline \multirow[t]{2}{*}{$\begin{array}{l}\text { Ramos et } \\
\text { al., } 2018^{43}\end{array}$} & $\begin{array}{l}\text { E: 12 } \\
(83.3 \%)\end{array}$ & 60.6 & $\begin{array}{l}\text { E: } 30.5 \pm \\
1.5\end{array}$ & NR & NR & Obesity & No & NR \\
\hline & $\begin{array}{l}\text { C: } 12 \\
(83.3 \%)\end{array}$ & & $\begin{array}{l}\text { C: } 33.1 \pm \\
2.8\end{array}$ & & & & & \\
\hline \multirow[t]{2}{*}{$\begin{array}{l}\text { Tanabe et } \\
\text { al., } 1989^{44}\end{array}$} & $\begin{array}{l}\text { E: } 21 \\
(52.4 \%)\end{array}$ & 48. 9 & NR & NR & NR & NR & No & NR \\
\hline & $\begin{array}{l}\text { C: } 10 \\
(50 \%)\end{array}$ & & & & & & & \\
\hline \multirow[t]{2}{*}{$\begin{array}{l}\text { Tsai et al., } \\
2002^{45}\end{array}$} & $\begin{array}{l}\text { E: } 12 \\
(41.7 \%)\end{array}$ & 47.9 & $\begin{array}{l}\text { E: } 26.1 \pm \\
4.5\end{array}$ & NR & Untrained & No & No & NR \\
\hline & $\begin{array}{l}\text { C: } 11 \\
(54.5 \%)\end{array}$ & & $\begin{array}{l}\text { C: } 25.0 \\
\pm 1.8\end{array}$ & & & & & \\
\hline \multirow[t]{2}{*}{$\begin{array}{l}\text { Tsai et al., } \\
2004^{46}\end{array}$} & $\begin{array}{l}\text { E: } 52 \\
(53.8 \%)\end{array}$ & 49. 1 & $\begin{array}{l}\text { E: } 23.6 \pm \\
1.8\end{array}$ & NR & Untrained & No & No & NR \\
\hline & $\begin{array}{l}\text { C: } 50 \\
(54 \%)\end{array}$ & & $\begin{array}{l}\text { C: } 23.8 \\
\pm 2.2\end{array}$ & & & & & \\
\hline
\end{tabular}

Note: E - exercise group. C - control group. CONT- continuous. INT - interval. NR- not reported. BMI- body mass index. DM2diabetes mellitus 2.**Soltani et al.,2019 and Tanaka et al., 1997 - Number of randomized individuals, because the number of analyzed was not reported in the study.

\subsection{Characteristics of interventions 3.3.1 General characteristics}


The general characteristics of the interventions is presented in Table 2. In the case of aerobic training, indoor cycling was the most reported modality (35.7\%), followed by running / walking on the treadmill (25\%) and soon after swimming (7.14\%), aquatic exercises $(7.14 \%)$ and dance (3.6\%). Regarding the methods applied to training, of the 28 aerobic training groups, only five (17.9\%) used interval training, while the other $23(82.1 \%)$ used continuous training. The total duration of the interventions ranged from 6 to 37 weeks and the sessions lasted from 20 to 60 minutes, performed 3 to 4 times a week. 
Table 2

Characteristics of the interventions.

\begin{tabular}{|c|c|c|c|c|c|c|}
\hline Study & $\begin{array}{l}\text { Intervention } \\
\text { period }\end{array}$ & Modality & Method & $\begin{array}{l}\text { Session } \\
\text { Duration }\end{array}$ & $\begin{array}{l}\text { Weekly } \\
\text { frequency }\end{array}$ & Intensity \\
\hline \multicolumn{7}{|c|}{ Progressive aerobic training } \\
\hline \multirow{2}{*}{$\begin{array}{l}\text { Abdelaal and } \\
\text { Mohamad, } \\
2014^{23}\end{array}$} & \multirow[t]{2}{*}{12 weeks } & \multirow[t]{2}{*}{$\begin{array}{l}\text { Treadmill (not defined whether } \\
\text { walking or running) }\end{array}$} & \multirow[t]{2}{*}{ Continuous } & $\begin{array}{l}\text { B: } 20- \\
35 \mathrm{~min}\end{array}$ & \multirow[t]{2}{*}{3} & $\begin{array}{l}\text { B: } 60- \\
65 \%\end{array}$ \\
\hline & & & & $\begin{array}{l}\mathrm{F}: 40- \\
50 \mathrm{~min}\end{array}$ & & $\begin{array}{l}F: 70- \\
75 \% \\
\text { HRmax }\end{array}$ \\
\hline \multirow[t]{2}{*}{$\begin{array}{l}\text { Baghaiee et al., } \\
2018^{24}\end{array}$} & \multirow[t]{2}{*}{12 weeks } & \multirow[t]{2}{*}{ NR } & \multirow[t]{2}{*}{ Continuous } & $\begin{array}{l}\text { B: } 25 \\
\text { min }\end{array}$ & \multirow[t]{2}{*}{3} & $\begin{array}{l}\text { B: } 50 \% \\
\text { HRmax }\end{array}$ \\
\hline & & & & $\begin{array}{l}\mathrm{F}: 45 \\
\mathrm{~min}\end{array}$ & & $\begin{array}{l}\text { F: } 70 \% \\
\text { HRmax }\end{array}$ \\
\hline \multirow[t]{2}{*}{$\begin{array}{l}\text { Farahani et al., } \\
2010^{25}\end{array}$} & \multirow[t]{2}{*}{10 weeks } & \multirow[t]{2}{*}{ Aquatic exercise } & \multirow[t]{2}{*}{ Continuous } & $35 \min$ & \multirow[t]{2}{*}{3} & $\begin{array}{l}\text { B: } 60- \\
65 \% \\
\text { HRmax }\end{array}$ \\
\hline & & & & & & $\begin{array}{l}\text { F: } 70- \\
75 \% \\
\text { HRmax }\end{array}$ \\
\hline \multirow{2}{*}{$\begin{array}{l}\text { Hagberg et al., } \\
1989^{26}\end{array}$} & \multirow[t]{2}{*}{37 weeks } & \multirow{2}{*}{$\begin{array}{l}\text { Walking/running (Treadmill)/ } \\
\text { Cycle Ergometer }\end{array}$} & \multirow[t]{2}{*}{ Continuous } & \multirow{2}{*}{$\begin{array}{l}45-60 \\
\min \end{array}$} & \multirow[t]{2}{*}{3} & B: NR \\
\hline & & & & & & $\begin{array}{l}\mathrm{F}: 85 \% \\
\mathrm{VO}_{2} \text { max }\end{array}$ \\
\hline \multirow{2}{*}{$\begin{array}{l}\text { Kokkinos et al., } \\
1995^{27}\end{array}$} & 16 weeks & \multirow[t]{2}{*}{ Cycle Ergometer } & \multirow[t]{2}{*}{ Continuous } & \multirow{2}{*}{$\begin{array}{l}20-60 \\
\min \end{array}$} & \multirow[t]{2}{*}{3} & \multirow{2}{*}{$\begin{array}{l}60-80 \% \\
\text { HRmax }\end{array}$} \\
\hline & 32 weeks & & & & & \\
\hline \multirow[t]{4}{*}{$\begin{array}{l}\text { Lamina et al., } \\
2010^{28}\end{array}$} & \multirow[t]{4}{*}{8 weeks } & \multirow[t]{4}{*}{ Cycle Ergometer } & \multirow[t]{2}{*}{ Continuous } & $\begin{array}{l}\text { B: } 45 \\
\text { min }\end{array}$ & \multirow[t]{2}{*}{ NR } & $\begin{array}{l}\text { B: } 60 \% \\
\text { HRmax }\end{array}$ \\
\hline & & & & $\begin{array}{l}\mathrm{F}: 60 \\
\mathrm{~min}\end{array}$ & & $\begin{array}{l}\text { F: } 79 \% \\
\text { HRmax }\end{array}$ \\
\hline & & & \multirow[t]{2}{*}{ Interval } & $\begin{array}{l}\text { B: } 45 \\
\min \end{array}$ & \multirow[t]{2}{*}{3} & $\begin{array}{l}60-79 \% \\
\operatorname{HRmax}\end{array}$ \\
\hline & & & & $\begin{array}{l}\mathrm{F}: 60 \\
\mathrm{~min}\end{array}$ & & \\
\hline \multirow[t]{2}{*}{$\begin{array}{l}\text { Latosik et al., } \\
2014^{29}\end{array}$} & 8 weeks & Nordic walking & Continuous & $45 \min$ & NR & $\begin{array}{l}\text { B:40- } \\
60 \% \\
\text { HRmax }\end{array}$ \\
\hline & & & & & & $\begin{array}{l}\text { F: } 38- \\
69 \% \\
\text { HRmax }\end{array}$ \\
\hline $\begin{array}{l}\text { Meirelles et al., } \\
2009^{30}\end{array}$ & 12 weeks & Walking/ running & Continuous & $40 \min$ & 3 & $\begin{array}{l}\text { B: } 75 \% \\
\text { HRmax }\end{array}$ \\
\hline & & & & & & $\begin{array}{l}\mathrm{F}: 85 \% \\
\text { HRmax }\end{array}$ \\
\hline
\end{tabular}

Note: $\mathrm{B}$ - beginning. $\mathrm{F}$ - final. Min - minutes. $\mathrm{HRmax}$ - maximum heart rate. $\mathrm{VO}_{2} \mathrm{max}$ - maximum volume of oxygen. $\mathrm{VO}_{2}$ peak - peak oxygen volume. HRres - heart rate reserve. - rating perceived exertion. 


\begin{tabular}{|c|c|c|c|c|c|c|}
\hline Study & $\begin{array}{l}\text { Intervention } \\
\text { period }\end{array}$ & Modality & Method & $\begin{array}{l}\text { Session } \\
\text { Duration }\end{array}$ & $\begin{array}{l}\text { Weekly } \\
\text { frequency }\end{array}$ & Intensity \\
\hline \multirow[t]{4}{*}{$\begin{array}{l}\text { Soltani et al., } \\
2019^{31}\end{array}$} & \multirow[t]{4}{*}{8 weeks } & \multirow[t]{4}{*}{ Walking/running (Treadmill) } & \multirow[t]{4}{*}{ Interval } & $27 \mathrm{~min}$ & \multirow[t]{4}{*}{3} & $\begin{array}{l}\mathrm{B}: 80 \% \\
\mathrm{VO}_{2} \text { peak }\end{array}$ \\
\hline & & & & & & $\begin{array}{l}\mathrm{F}: 100 \% \\
\mathrm{VO}_{2} \text { peak }\end{array}$ \\
\hline & & & & $32 \mathrm{~min}$ & & $\begin{array}{l}\mathrm{B}: 75 \% \\
\mathrm{VO}_{2} \text { peak }\end{array}$ \\
\hline & & & & & & $\begin{array}{l}\mathrm{F}: 90 \mathrm{VO}_{2} \\
\text { peak }\end{array}$ \\
\hline \multirow[t]{2}{*}{$\begin{array}{l}\text { Tanaka et al., } \\
1997^{32}\end{array}$} & \multirow[t]{2}{*}{10 weeks } & \multirow[t]{2}{*}{ Swimming } & \multirow[t]{2}{*}{ Continuous } & $\begin{array}{l}\text { B: } 30 \\
\text { min }\end{array}$ & \multirow[t]{2}{*}{3} & \multirow[t]{2}{*}{$\begin{array}{l}60 \% \\
\text { HRres }\end{array}$} \\
\hline & & & & $\begin{array}{l}\mathrm{F}: 45 \\
\mathrm{~min}\end{array}$ & & \\
\hline \multirow[t]{2}{*}{$\begin{array}{l}\text { Turner et al., } \\
2000^{33}\end{array}$} & \multirow[t]{2}{*}{28 weeks } & \multirow[t]{2}{*}{$\begin{array}{l}\text { Walking/running / Cycle } \\
\text { Ergometer }\end{array}$} & \multirow[t]{2}{*}{ Continuous } & $\begin{array}{l}30-50 \\
\min \end{array}$ & \multirow[t]{2}{*}{4} & $\begin{array}{l}\text { B: } 60- \\
70 \% \\
\text { HRmax }\end{array}$ \\
\hline & & & & & & $\begin{array}{l}\text { F: 70- } \\
80 \% \\
\text { HRmax }\end{array}$ \\
\hline \multirow[t]{2}{*}{$\begin{array}{l}\text { Wong et al., } \\
2018^{34}\end{array}$} & \multirow[t]{2}{*}{20 weeks } & \multirow[t]{2}{*}{ Swimming } & \multirow[t]{2}{*}{ Continuous } & $\begin{array}{l}\text { B: } 25- \\
30 \text { min }\end{array}$ & \multirow[t]{2}{*}{3 to 4} & $\begin{array}{l}\text { B: } 60 \% \\
\text { HRmax }\end{array}$ \\
\hline & & & & $\begin{array}{l}\mathrm{F}: 40- \\
45 \mathrm{~min}\end{array}$ & & $\begin{array}{l}\text { F: } 70- \\
75 \% \\
\text { HRmax }\end{array}$ \\
\hline \multicolumn{7}{|c|}{ Non-progressive aerobic training } \\
\hline \multirow{2}{*}{$\begin{array}{l}\text { Arca et al., } \\
2014^{35}\end{array}$} & \multirow[t]{2}{*}{12 weeks } & Cycle Ergometer & \multirow[t]{2}{*}{ Continuous } & \multirow[t]{2}{*}{$20 \mathrm{~min}$} & \multirow[t]{2}{*}{3} & \multirow{2}{*}{$\begin{array}{l}50-60 \% \\
\text { HRres }\end{array}$} \\
\hline & & Aquatic exercise & & & & \\
\hline He et al., $2018^{36}$ & 12 weeks & Walking & Continuous & $60 \mathrm{~min}$ & 3 & $\begin{array}{l}45-50 \% \\
\mathrm{VO}_{2} \mathrm{max}\end{array}$ \\
\hline $\begin{array}{l}\text { Hong et al., } \\
2018^{37}\end{array}$ & 12 weeks & Walking/running (Treadmill) & Continuous & $60 \mathrm{~min}$ & 4 & $\begin{array}{l}60 \% \\
\mathrm{VO}_{2} \max \end{array}$ \\
\hline $\begin{array}{l}\text { Khalid et al., } \\
2013^{38}\end{array}$ & 8 weeks & Walking (Treadmill) & Continuous & $20 \min$ & 3 & $\begin{array}{l}60-75 \% \\
\operatorname{HRmax}\end{array}$ \\
\hline $\begin{array}{l}\text { Izadi et al., } \\
2017^{39}\end{array}$ & 6 weeks & Cycle Ergometer & Interval & $35 \mathrm{~min}$ & 3 & $\begin{array}{l}85-90 \% \\
\text { HRres }\end{array}$ \\
\hline $\begin{array}{l}\text { Koga et al., } \\
1992^{40}\end{array}$ & 10 weeks & Cycle Ergometer & Continuous & $60 \mathrm{~min}$ & 3 & $\begin{array}{l}50 \% \\
\mathrm{VO}_{2} \max \end{array}$ \\
\hline $\begin{array}{l}\text { Maruf et al., } \\
2014^{41}\end{array}$ & 12 weeks & Dance & Interval & $35 \mathrm{~min}$ & 3 & $\begin{array}{l}50-70 \% \\
\text { HRres }\end{array}$ \\
\hline $\begin{array}{l}\text { Miura et al., } \\
1994^{42}\end{array}$ & 10 weeks & Cycle Ergometer & Continuous & $60 \mathrm{~min}$ & 3 & $\begin{array}{l}40-60 \% \\
\mathrm{VO}_{2} \mathrm{max}\end{array}$ \\
\hline
\end{tabular}

Note: $\mathrm{B}$ - beginning. $\mathrm{F}$ - final. Min - minutes. $\mathrm{HRmax}$ - maximum heart rate. $\mathrm{VO}_{2} \mathrm{max}$ - maximum volume of oxygen. $\mathrm{VO}_{2}$ peak - peak oxygen volume. HRres - heart rate reserve. - rating perceived exertion. 


\begin{tabular}{|c|c|c|c|c|c|c|}
\hline Study & $\begin{array}{l}\text { Intervention } \\
\text { period }\end{array}$ & Modality & Method & $\begin{array}{l}\text { Session } \\
\text { Duration }\end{array}$ & $\begin{array}{l}\text { Weekly } \\
\text { frequency }\end{array}$ & Intensity \\
\hline $\begin{array}{l}\text { Ramos et al., } \\
2018^{43}\end{array}$ & 12 weeks & $\begin{array}{l}\text { Athletics Track (not defined } \\
\text { whether walking or running) }\end{array}$ & Continuous & $50 \mathrm{~min}$ & 3 & $\begin{array}{l}60 \% \\
\text { HRmax/ } \\
4-6 \text { RPE } \\
\text { (OMNI) }\end{array}$ \\
\hline $\begin{array}{l}\text { Tanabe et al., } \\
1989^{44}\end{array}$ & 10 weeks & Cycle Ergometer & Continuous & $60 \min$ & 3 & $\begin{array}{l}40-60 \% \\
\mathrm{VO}_{2} \mathrm{max}\end{array}$ \\
\hline Tsai et al., $2002^{45}$ & 12 weeks & Walking/ (Treadmill) & Continuous & $30 \mathrm{~min}$ & 3 & $\begin{array}{l}60-70 \% \\
\text { HRres }\end{array}$ \\
\hline Tsai et al., $2004^{46}$ & 10 weeks & Walking/running (Treadmill) & Continuous & $30 \mathrm{~min}$ & 3 & $\begin{array}{l}60-70 \% \\
\text { HRres }\end{array}$ \\
\hline
\end{tabular}

\subsubsection{Progressive aerobic training}

Regarding aerobic training with progression, 15 exercise groups ${ }^{23-34}$ were analyzed, with the majority (80\%) of the studies applying continuous training protocols. The total duration of interventions ranged from 8 to 37 weeks and the duration of sessions from 20 to 60 minutes, with two studies ${ }^{28,32}$ that progressed only in duration not showing the number of progressions made. Regarding the weekly frequency of training sessions, one study ${ }^{33}$ reported four weekly sessions, 11 exercise groups ${ }^{23-28,30-32}$ had three weekly sessions, one exercise group ${ }^{34}$ had a frequency of three to four weekly sessions and two ${ }^{28,29}$ did not report this information. No study reported progression in weekly frequency. Regarding intensity, seven exercise groups ${ }^{25,26,29-31,33}$ had only progression for this variable, with the most widely used method for prescribing the maximum heart rate (HRmax), applied in 11 exercise groups ${ }^{23-25,27-30,33,34}$, followed by peak oxygen consumption (VO2peak), maximum oxygen consumption (VO2max) and reserve heart rate (HRres).

\subsubsection{Non-progressive aerobic training}

Regarding aerobic training without progression, 13 aerobic exercise groups ${ }^{35-46}$ were analyzed, of which only two (15.4\%) used interval training ${ }^{39,41}$. A single study showed a frequency of four training sessions per week ${ }^{37}$, while all others used three sessions per week. The total duration of the interventions ranged from 6 to 12 weeks and the duration of the sessions ranged from 20 to 60 minutes. For intensity, the most used method for prescription was HRres, applied in six studies $35,39,41,45,46$, followed by V02max, HRmax and rating of perceived exertion (RPE).

\subsection{Analysis of the risk of bias}

Among all the included studies, only $16.7 \%$ carried out the process of randomization and allocation confidentiality of the participants in the groups in an appropriate manner, $75 \%$ did not provide enough information to determine the randomization process and almost $80 \%$ failed to provide details regarding allocation secrecy. Still, only $25 \%$ of the studies were carried out with blinded evaluators, $58.3 \%$ of the studies described sample losses and $20.8 \%$ adopted analysis by intention to treat. Data on risk of bias separated by group with and without progression can be seen in Table 3. 
Table 3

Risk of bias

\begin{tabular}{|c|c|c|c|c|c|}
\hline Study & $\begin{array}{l}\text { Random } \\
\text { sequence } \\
\text { generation }\end{array}$ & $\begin{array}{l}\text { Allocation } \\
\text { concealment }\end{array}$ & $\begin{array}{l}\text { Blinding of } \\
\text { outcome } \\
\text { assessment }\end{array}$ & $\begin{array}{l}\text { Description of } \\
\text { sample losses }\end{array}$ & $\begin{array}{l}\text { Intention-to-treat } \\
\text { analysis }\end{array}$ \\
\hline \multicolumn{6}{|l|}{ Progressive aerobic training } \\
\hline $\begin{array}{l}\text { Abdelaal and Mohamad, } \\
2014^{23}\end{array}$ & Low & Low & Low & Low & Low \\
\hline Baghaiee et al., $2018^{24}$ & Unclear & Unclear & Unclear & High & Unclear \\
\hline Farahani et al., $2010^{25}$ & High & Unclear & Unclear & High & Unclear \\
\hline Hagberg et al., $1989^{26}$ & Unclear & Unclear & Unclear & Low & Unclear \\
\hline Kokkinos et al., $1995^{27}$ & Unclear & Unclear & Unclear & Low & High \\
\hline Lamina et al., $2010^{28}$ & Unclear & Unclear & Low & Low & High \\
\hline Latosik et al., $2014^{29}$ & Unclear & Unclear & Unclear & Low & High \\
\hline Meirelles et al., $2009^{30}$ & Unclear & Unclear & Unclear & High & Low \\
\hline Soltani et al., $2019^{31}$ & Unclear & Unclear & Unclear & Low & Unclear \\
\hline Tanaka et al., $1997^{32}$ & Unclear & Unclear & Low & High & Unclear \\
\hline Turner et al., $2000^{33}$ & High & High & Unclear & Low & Unclear \\
\hline Wong et al., $2018^{34}$ & Low & Low & Low & Low & Low \\
\hline \multicolumn{6}{|c|}{ Non-progressive aerobic training } \\
\hline Arca et al., $2014^{35}$ & Unclear & Unclear & Unclear & High & Unclear \\
\hline He et al., $2018^{36}$ & Unclear & Unclear & Unclear & High & High \\
\hline Hong et al., $2018^{37}$ & Unclear & Unclear & Unclear & High & Unclear \\
\hline Khalid et al., $2013^{38}$ & Low & Low & Low & Low & High \\
\hline Izadi et al., $2017^{39}$ & Unclear & Unclear & Unclear & Low & High \\
\hline Koga et al., $1992^{40}$ & Unclear & Unclear & High & High & Unclear \\
\hline Maruf et al., $2014^{41}$ & Low & Unclear & Unclear & Low & Low \\
\hline Miura et al., $1994^{42}$ & Unclear & Unclear & Unclear & Low & Unclear \\
\hline Ramos et al., $2018^{43}$ & Unclear & Low & Unclear & High & Unclear \\
\hline Tanabe et al., $1989^{44}$ & Unclear & Unclear & Unclear & High & Low \\
\hline Tsai et al., $2002^{45}$ & Unclear & Unclear & Unclear & Low & High \\
\hline Tsai et al., $2004^{46}$ & Unclear & Unclear & Low & Low & High \\
\hline
\end{tabular}




\subsubsection{Effect of aerobic training (SBP and DBP)}

The aerobic training analyzed in general, totaling 716 included participants, demonstrated a reduction in SBP with a magnitude of $10.56 \mathrm{mmHg}(95 \% \mathrm{Cl}-14.083,-7.026 ; \mathrm{p}<0.001 ; 2$ : $98 \%)$ and in DBP of $5.84 \mathrm{mmHg}(95 \% \mathrm{Cl}-8.226,-3.465 ; \mathrm{p}<$ $0.001 ;$ : $:$ : 97\%).

\subsubsection{Effect of progressive aerobic training (SBP and DBP)}

The results related to aerobic training with progression were analyzed in 15 studies, showing a reduction in SBP of 10.67 $\mathrm{mmHg}(95 \% \mathrm{Cl}-15.421,-5.926 ; \mathrm{p}<0.001 ;$ P: $99 \%)$ and in DBP of $5.49 \mathrm{mmHg}(95 \% \mathrm{Cl}-8.663,-2.310 ; \mathrm{p}<0.001 ;$ P: 99\%) (Fig. 2). 3.5.3 Effect of different progressions (SBP and DBP)

Analyzing only the studies that progressed in intensity, there was a decrease in SBP of $12.89 \mathrm{mmHg}(95 \% \mathrm{Cl}-20.134,-5.648$; $\mathrm{p}<0.001 ; P: 64 \%)$ and in DBP of $7.09 \mathrm{mmHg}(95 \% \mathrm{Cl}-11.707,-2.478 ; \mathrm{p}=0.003 ; 2: 69 \%)$, while for progression only in duration, a reduction in SBP of $13.98 \mathrm{mmHg}$ was found $(95 \% \mathrm{Cl}-24.238,-3.716 ; \mathrm{p}=0.008 ; P: 36 \%)$ and in DBP $5.07 \mathrm{mmHg}$ $(95 \% \mathrm{Cl}-7.288,-2.843 ; \mathrm{p}<0.001 ; P: 0 \%)$. When analyzing the progression in the intensity and duration variables together, statistically significant reductions were found only in the SBP $(-8.28 \mathrm{mmHg} ; 95 \% \mathrm{Cl}-15.089,-1.479 ; \mathrm{p}=0.017 ; P: 100 \%)$. In DBP, the reduction was $4.48 \mathrm{mmHg}$, without statistical significance $\left(95 \% \mathrm{Cl}-9.100,0.132 ; \mathrm{p}=0.057 ; P^{2}: 99 \%\right)$.

\subsubsection{Effect of non-progressive aerobic training (SBP and DBP)}

Regarding the effect of aerobic training without progression, adopted in 13 studies, a reduction was found in SBP of 10.17 $\mathrm{mmHg}\left(95 \% \mathrm{Cl}-12.213,-8.120 ; \mathrm{p}<0.001 ; P^{2}: 0 \%\right)$ and in DBP of $6.51 \mathrm{mmHg}(95 \% \mathrm{Cl}-9.147,-3.868 ; \mathrm{p}<0.001 ;$ P: 61\%) (Fig. 3).

\subsubsection{Effect of aerobic training on subgroups (SBP and DBP)}

Subgroup analyses show the effects of aerobic training on SBP and DBP separately under different conditions, between female and male, between subjects with and without comorbidities, between subjects with and without the use of antihypertensive drugs, between different weekly durations of intervention, between methods (continuous and interval), between different modalities and between aquatic and terrestrial environment.

Among the subgroups, in absolute terms, aerobic training provided the greatest reduction magnitude both in SBP (-12.01 $\left.\mathrm{mmHg} ; 95 \% \mathrm{Cl}-12.56,-11.46 ; \mathrm{p} \varangle 0.001 ; I^{2}: 0 \%\right)$ and in DBP $\left(-7.94 \mathrm{mmHg} ; 95 \% \mathrm{Cl}-10.58,-5.29 ; \mathrm{p} \otimes 0.001 ; I^{2}: 15 \%\right)$ when the exercise was performed in the aquatic environment. The lowest magnitude of SBP reduction after aerobic training was observed in those individuals who did not use any antihypertensive medication $(-8.18 \mathrm{mmHg} ; 95 \% \mathrm{Cl}-14.58,-1.78 ; p=0.012$; 1: $99 \%)$. For DBP, the lowest magnitude of reduction after aerobic training was observed in the subgroup of men (-2.80 $\mathrm{mmHg} ; 95 \% \mathrm{Cl}-4.76,-0.85 ; \mathrm{p}=0.005 ;$ I: $76 \%$ ) (Table 4). 
Meta-analysis results.

\begin{tabular}{|c|c|c|c|c|c|c|c|c|c|}
\hline \multirow{3}{*}{ Sub-analysis } & \multirow{3}{*}{$\mathbf{N}$} & \multicolumn{4}{|c|}{ Systolic blood pressure } & \multicolumn{4}{|c|}{ Diastolic blood pressure } \\
\hline & & \multirow{2}{*}{$\begin{array}{l}\text { Mean difference } \\
\text { (Cl 95\%) }\end{array}$} & \multirow{2}{*}{$\begin{array}{l}p \\
\text { value }\end{array}$} & \multicolumn{2}{|c|}{ Heterogeneity } & \multirow{2}{*}{$\begin{array}{l}\text { Mean difference } \\
\text { (Cl 95\%) }\end{array}$} & \multirow{2}{*}{$\mathrm{p}_{\text {value }}$} & \multicolumn{2}{|c|}{ Heterogeneity } \\
\hline & & & & $1^{2}$ & $\begin{array}{l}p \\
\text { value }\end{array}$ & & & $I^{2}$ & $\mathrm{p}_{\text {value }}$ \\
\hline \multicolumn{10}{|l|}{ Sex } \\
\hline Men & 9 & $\begin{array}{l}-9.89(-17.11 \\
-2.67)\end{array}$ & 0.007 & $89 \%$ & $<.001$ & $-2.80(-4.76 ;-0.85)$ & 0.005 & $76 \%$ & $<.001$ \\
\hline Women & 7 & $\begin{array}{l}-11.98(-12.52 \\
-11.43)\end{array}$ & $<0.001$ & $0 \%$ & 0.471 & $-5.81(-9.36 ;-2.26)$ & 0.001 & $75 \%$ & $<0.001$ \\
\hline \multicolumn{10}{|l|}{ Comorbidities } \\
\hline $\begin{array}{l}\text { With } \\
\text { comorbidities }\end{array}$ & 6 & $\begin{array}{l}-10.17(-13.56 \\
-6.79)\end{array}$ & $<0.001$ & $41 \%$ & 0.132 & $\begin{array}{l}-7.76(-11.14 \\
-4.37)\end{array}$ & $<.001$ & $67 \%$ & 0.010 \\
\hline $\begin{array}{l}\text { Without } \\
\text { comorbidities }\end{array}$ & 15 & $\begin{array}{l}-10.46(-13.10 \\
-7.83)\end{array}$ & $<.001$ & $28 \%$ & 0.153 & $-4.59(-6.57 ;-2.61)$ & $<.001$ & $64 \%$ & $<.001$ \\
\hline \multicolumn{10}{|l|}{$\begin{array}{l}\text { Antihypertensive } \\
\text { drugs }\end{array}$} \\
\hline User & 10 & $\begin{array}{l}-10.47(-14.63 \\
-6.31)\end{array}$ & $<.001$ & $63 \%$ & 0.004 & $-6.44(-9.95 ;-2.93)$ & $<.001$ & $80 \%$ & $<.001$ \\
\hline Non-user & 8 & $\begin{array}{l}-8.18(-14.58 \\
-1.78)\end{array}$ & 0.012 & $99 \%$ & $<.001$ & $-6.45(-11.2 ;-1.65)$ & 0.008 & $99 \%$ & $\dot{0} 001$ \\
\hline \multicolumn{10}{|l|}{$\begin{array}{l}\text { Intervention } \\
\text { duration }\end{array}$} \\
\hline$<12$ weeks & 13 & $\begin{array}{l}-11.37(-14.67 \\
-8.08)\end{array}$ & $<.001$ & $34 \%$ & 0.110 & $-5.04(-7.50 ;-2.57)$ & $<.001$ & $71 \%$ & $<.001$ \\
\hline 12 to 24 weeks & 12 & $\begin{array}{l}-10.80(-16.01 \\
-5.58)\end{array}$ & $<001$ & $99 \%$ & $<0.001$ & $\begin{array}{l}-6.37(-10.23 \\
-2.52)\end{array}$ & 0.001 & $99 \%$ & $<0.001$ \\
\hline > 24 weeks & 3 & $\begin{array}{l}-8.73(-15.84 \\
-1.62)\end{array}$ & 0.016 & $0 \%$ & 0.546 & $\begin{array}{l}-6.37(-10.81 \\
-1.93)\end{array}$ & 0.005 & $0 \%$ & 0.842 \\
\hline \multicolumn{10}{|l|}{ Method } \\
\hline Continuous & 23 & $\begin{array}{l}-10.62(-14.52 \\
-6.71)\end{array}$ & $<0.001$ & $99 \%$ & $<.001$ & $-6.10(-8.78 ;-3.42)$ & $<.001$ & $98 \%$ & $<.001$ \\
\hline Interval & 5 & $\begin{array}{l}-10.32(-16.08 \\
-4.56)\end{array}$ & $<.001$ & $48 \%$ & 0.101 & $-4.62(-6.43 ;-2.82)$ & $<.001$ & $0 \%$ & 0.686 \\
\hline \multicolumn{10}{|l|}{ Modality } \\
\hline Walking/running & 11 & $\begin{array}{l}-11.20(-14.61 \\
-7.78)\end{array}$ & $<.001$ & $66 \%$ & 0.001 & $\begin{array}{l}-7.65(-10.42 \\
-4.88)\end{array}$ & $<.001$ & $81 \%$ & $<.001$ \\
\hline Cycle ergometer & 9 & $\begin{array}{l}-10.89(-15.76 \\
-6.02)\end{array}$ & $<.001$ & $51 \%$ & 0.038 & $-4.01(-6.31 ;-1.71)$ & $<.001$ & $55 \%$ & 0.022 \\
\hline $\begin{array}{l}\text { Various } \\
\text { modalities }\end{array}$ & 2 & $\begin{array}{l}-11.12(-20.24 \\
-2.01)\end{array}$ & 0.017 & $0 \%$ & 0.466 & $\begin{array}{l}-7.61(-13.74 \\
-1.48)\end{array}$ & 0.015 & $0 \%$ & 0.911 \\
\hline \multicolumn{10}{|l|}{ Training location } \\
\hline Number of a & es. & $5 \%$ - confidenc & al. $l^{2}$ & $\mathrm{ca}$ & e p & tage of heteroge & & & \\
\hline
\end{tabular}




\begin{tabular}{|c|c|c|c|c|c|c|c|c|c|}
\hline \multirow[b]{2}{*}{ Dry-land } & \multirow[b]{2}{*}{23} & \multicolumn{4}{|c|}{ Systolic blood pressure } & \multicolumn{4}{|c|}{ Diastolic blood pressure } \\
\hline & & $\begin{array}{l}-11.06(-13.53 \\
-8.59)\end{array}$ & $<.001$ & $54 \%$ & 0.001 & $-6.23(-8.25 ;-4.22)$ & $\dot{0} 001$ & $82 \%$ & $<.001$ \\
\hline Aquatic & 4 & $\begin{array}{l}-12.01(-12.56 ; \\
-11.46)\end{array}$ & $\begin{array}{l}< \\
0.001\end{array}$ & $0 \%$ & 0.666 & $\begin{array}{l}-7.94(-10.58 \\
-5.29)\end{array}$ & $\begin{array}{l}< \\
0.001\end{array}$ & $15 \%$ & 0.315 \\
\hline
\end{tabular}

\subsubsection{Meta-regression}

According to the results of the meta-regression analyses, only age showed an association with the reduction of SBP ( $\beta$ : $-0.323 ; \mathrm{Cl}-0.339,-0.307 ; \mathrm{p}<0.001)$, it being considered a predictor in reducing this variable as a result of aerobic training. Thus, the older the sample, the greater the reduction in SBP after the aerobic training period. The variables BMI, number of antihypertensive users, baseline SBP, weekly training frequency, weekly duration and intervention period were not associated with SBP reduction. No variable was associated with a reduction in DBP due to aerobic training (Table 5).

Table 5

Meta-regression results

\begin{tabular}{|c|c|c|c|c|c|c|c|}
\hline \multirow[t]{2}{*}{ Moderator } & \multirow{2}{*}{$\begin{array}{l}\text { Number of } \\
\text { studies }\end{array}$} & \multicolumn{3}{|c|}{ Systolic blood pressure } & \multicolumn{3}{|c|}{ Diastolic blood pressure } \\
\hline & & B & $\mathrm{Cl} 95 \%$ & $\begin{array}{l}\mathrm{p}- \\
\text { value }\end{array}$ & B & $\mathrm{Cl} 95 \%$ & p-value \\
\hline Mean age (years) & 23 & -0.323 & $\begin{array}{l}-0.339 \\
-0.307\end{array}$ & $<001$ & -0.121 & $\begin{array}{l}-0.337 \\
0.095\end{array}$ & 0.273 \\
\hline Body mass index $\left(\mathrm{kg} / \mathrm{m}^{2}\right)$ & 21 & 0.192 & $-0.645 ; 1.028$ & 0.653 & -0.269 & $\begin{array}{l}-0.827 \\
0.288\end{array}$ & 0.344 \\
\hline $\begin{array}{l}\text { Antihypertensives users } \\
\text { (n) }\end{array}$ & 19 & -0.028 & $-0.248 ; 0.193$ & 0.805 & 0.071 & $\begin{array}{l}-0.105 \\
0.247\end{array}$ & 0.428 \\
\hline SBP basal (mmHg) & 28 & -0.234 & $-0.482 ; 0.014$ & 0.065 & - & - & - \\
\hline DBP basal (mmHg) & 28 & - & - & - & -0.116 & $\begin{array}{l}-0.453 \\
0.220\end{array}$ & 0.498 \\
\hline Weekly frequency & 26 & -1.407 & $-9.057 ; 6.242$ & 0.718 & -1.525 & $\begin{array}{l}-6.791 \\
3.741\end{array}$ & 0.570 \\
\hline Weekly length (min) & 27 & 0.016 & $-0.045 ; 0.077$ & 0.604 & 0.018 & $\begin{array}{l}-0.022 \\
0.059\end{array}$ & 0.368 \\
\hline $\begin{array}{l}\text { Intervention period } \\
\text { (weeks) }\end{array}$ & 28 & 0.107 & $-0.295 ; 0.508$ & 0.603 & -0.114 & $\begin{array}{l}-0.366 \\
0.138\end{array}$ & 0.376 \\
\hline
\end{tabular}

\section{Discussion}

This systematic review with meta-analysis aimed to analyze the effects of aerobic training, with and without progression, on the SBP and DBP of hypertensive adults. Our main results show that both aerobic training strategies (with and without progression) were effective in reducing BP and that older age is a factor associated with greater BP reductions due to aerobic training in hypertensive individuals.

The general results of the present study (aerobic training vs. control group) are in line with results from other meta-analyses, which showed an average reduction of 8 to $12 \mathrm{mmHg}$ in SBP and 5 to $6 \mathrm{mmHg}$ in DBP in hypertensive adults ${ }^{11-13}$. Thus, our results reinforce the importance of aerobic training as a primary strategy for the treatment of hypertension, since the reduction in $\mathrm{BP}$ resulting from this practice is similar to that achieved with treatment with antihypertensive drugs ${ }^{47}$. These 
results are clinically relevant since a $10 \mathrm{mmHg}$ decrease in SBP is associated with a $20 \%$ reduction in the risk of developing cardiovascular disease, $27 \%$ in the occurrence of stroke and $13 \%$ in the risk of mortality ${ }^{48}$.

Although there is no difference in the BP reduction between aerobic training with and without progression, reductions of greater magnitudes occurred in studies that progressed in the duration and intensity variables separately. The studies that progressed in intensity 25,26,29-31,33 showed reductions of $12.89 \mathrm{mmHg}(\mathrm{SBP})$ and $7.09 \mathrm{mmHg}$ (DBP) and achieved the highest percentages of intensity compared to the other studies. Studies that progressed in duration ${ }^{28,32}$, on the other hand, showed reductions of $13.98 \mathrm{mmHg}$ in SBP and $5.07 \mathrm{mmHg}$ in DBP, and achieved the longest durations in comparison to all studies with and without progression. The studies that progressed in both $23,24,27,28,34$ showed more modest reductions in SBP $(8.28 \mathrm{mmHg})$, with no significant reduction in DBP, and achieved session durations longer than the other studies, however the percentage of intensity was below that observed in general, both in with progression and without progression studies. Although the greatest reductions in SBP occur with studies that have progressed in duration, it is possible that the intensity of training is an important modulator of BP reduction, since both studies that have progressed in duration and those that have progressed in intensity have achieved or maintained a high intensity of training. In addition, our results showed a response of greater magnitude in studies with progression in duration, for SBP, and in intensity, for DBP, which may be associated with different mechanisms of action. Bearing in mind that the performance of these mechanisms in response to training is still unknown ${ }^{49}$, it is not possible to state the reason associated with these differences.

The literature points to exercise intensity as a determining factor for BP changes in response to training programs ${ }^{7,50}$, indicating a dose-response relationship, so that higher intensities of trainig promote greater reductions in $\mathrm{BP}^{51}$. As for the duration of the session, there seems to be no dose-response relationship, as longer durations do not necessarily indicate greater reductions in BP, with beneficial effects occurring even with shorter periods of exercise ${ }^{52}$. However, although some studies have investigated the effects of different intensities and durations of exercise, there is insufficient evidence to determine the relationship of these variables with the BP response ${ }^{53}$, especially when not analyzed as isolated doses, but in relation to their manipulations throughout interventions. In the general analysis of our results, the greatest reduction occurred in the study by Meirelles et al. (2009) ${ }^{30}$, both for SBP $(-26.3 \mathrm{mmHg})$ and for DBP $(-13.4 \mathrm{mmHg})$, having reported progression in intensity and reached high intensity ( $85 \% \mathrm{HRmax}$ ). In addition, although there was no progression in duration, the length of the sessions was intermediate when compared to the other studies (40 minutes).

Although the evidence regarding progression is not concrete, the manipulation of training variables, such as duration and intensity, are recommended, and must respect a gradual process ${ }^{20}$, especially in intensity progression ${ }^{50}$. This strategy, in addition to reducing the risks of musculoskeletal injuries and cardiovascular events, favors greater participation by the participant in training ${ }^{20}$. In addition, other precautions must be considered in this process, such as the levels of BP the person has, recent changes in antihypertensive drugs, effects caused by exercise and medications, in addition to the presence of other diseases and related conditions ${ }^{50}$.

With regard to the subgroup analysis by sex, the effects of the interventions were effective for both men and women, and, in absolute terms, the magnitude of reduction observed for women was greater in SBP $(11.98 \mathrm{mmHg})$ and DBP $(5.81 \mathrm{mmHg})$, although there was no statistical difference between the groups. Turnbull et al. ${ }^{54}$, in a meta-analysis, demonstrated that men and women have BP reductions of similar magnitudes, thus demonstrating that training methods do not need to differ between sexes. For analysis between practitioners with and without comorbidities, both groups showed significant BP reductions, with similar magnitudes. This finding demonstrates great clinical relevance, considering that hypertensive patients with comorbidities, which present more serious health risks and greater chances of developing coronary artery disease $(C A D)^{55}$, benefit from the training as much as those without comorbidities.

Regarding the results by users and nonusers of antihypertensive drugs, both showed significant and similar reductions in SBP and DBP, emphasizing that aerobic training also has great hypotensive potential, being able to further optimize the treatment of hypertension, reducing the risks of complications and improving the clinical picture of hypertensive patients ${ }^{56}$. 
Reinforcing these results, the meta-regression analysis showed that the use of antihypertensive drugs was not a moderator in reducing BP during aerobic training. However, different dosages and classes of antihypertensive drugs were used in the studies included in this review, which makes it difficult to understand the effects of these factors on the pressure responses observed. Similar to our results, meta-analysis by Sardeli et al. ${ }^{57}$ also found that medication use did not affect BP reduction in response to training. However, this result was not specific to aerobic training and included few studies. Studies evaluating the effects of drug therapy and exercise on BP variables mainly focus on outcomes separately and have conflicting results. Thus, the evidence regarding the interaction between the use of medications and pressure responses to exercise is still scarce $^{53}$.

With regard to the duration of the interventions, it was shown that regardless of the aerobic training being performed for short or long periods ( $<12$ weeks, 12-24 weeks and $>24$ weeks), the BP reductions are similar. Likewise, a systematic review by Cao et al. ${ }^{13}$ demonstrated that interventions of less than 8 weeks, between 8 and 12 weeks and of more than 12 weeks were similarly effective in decreasing BP. On the other hand, Cornelissen and Smart (2013) ${ }^{11}$ observed that aerobic training periods of less than 12 weeks produced greater reductions in SBP and DBP when compared to longer periods, which could be related to the greater adherence of participants to shorter. However, we should consider the importance of the continuity of the training, in order to maintain the benefits achieved. Considering the divergence of the available findings, there is a need for more studies with good methodological quality to better understand the role of the duration of the intervention in the nondrug treatment of hypertensive patients.

As for the training methods, the continuous and the interval methods promoted similar reductions in SBP $(-10.62 \mathrm{mmHg}$; $-10.32 \mathrm{mmHg}$, respectively) and DBP $(-6.10 \mathrm{mmHg} ;-4.62 \mathrm{mmHg}$, respectively), showing that both are effective. Another recent meta-analysis, carried out with the hypertensive population ${ }^{58}$, showed reductions in SBP for both training methods when compared to control groups (continuous: $-3.70 \mathrm{mmHg}$; interval: $-5.64 \mathrm{mmHg}$ ), but without difference between training groups. For DBP, reduction was also found after continuous $(-2.41 \mathrm{mmHg})$ and interval $(-4.80 \mathrm{mmHg})$ training when compared to control groups, but the magnitude of DBP reduction in the interval method was significantly greater when compared to the continuous one.

Regarding the training modality, our study shows that the SBP and DBP reduce in a similar way regardless of whether the aerobic exercise is performed with walking / running $(-11.20 \mathrm{mmHg})$, on cycle ergometers $(-10.89 \mathrm{mmHg})$ or combining the training modalities $(-11.12 \mathrm{mmHg})$. This finding has an important practical application, as it demonstrates that exercise professionals can choose the form of aerobic exercise according to the patient's preference, thus being able to favor adherence due to the ease of access to a certain modality or to specific clinical conditions (i.e., using a cycle ergometer due to difficulty mobility with support of their own weight), without prejudice to the reduction in BP.

As for the training location, conducting training in water may be an alternative for the hypertensive population, as it has also shown slightly higher magnitudes of BP reductions (SBP - terrestrial: $-11.06 \mathrm{mmHg}$; aquatic: $-12.01 \mathrm{mmHg}$; DBP - terrestrial: $-6.23 \mathrm{mmHg}$; aquatic: $-7.94 \mathrm{mmHg}$ ) in absolute terms, without statistical differences. Another study of systematic review also observed that training in water reduces SBP and DBP in a similar way to terrestrial training in adults and the elderly, with $54.5 \%$ of the sample classified as hypertensive and $27.3 \%$ with pre-hypertensive ${ }^{59}$. It is noteworthy that studies comparing exercises performed in different media and evaluating different outcomes in hypertensive patients are still scarce, especially in the case of chronic effects.

The meta-regression analysis showed a significant association only for age and SBP responses, indicating that older individuals showed greater magnitude reductions in SBP after aerobic training. Since the prevalence of hypertension is higher at older ages ${ }^{60}$, the results of the present study suggest that the practice of aerobic training is an important nondrug strategy for reducing BP in the elderly, which has been observed previously ${ }^{61}$. However, our results were different from those evidenced by previous studies $11,57,62$. There is still disagreement in the literature regarding the influence of age on the effects of BP reduction in response to aerobic training, so that other factors must also be considered.

Page $17 / 24$ 
An important point to be highlighted in the present study is that most studies analyzed with progression used HRmax to prescribe the intensity of exercise $\mathrm{e}^{23-25,27-30,33,34}$ while only one study without progression used this method ${ }^{38}$. Since HRmax is a marker with limitations, we can explain, in part, why the studies that performed progression did not find greater chronic reductions in BP, since the prescribed intensity may have been underestimated in these studies. That is, not progressing violates a training principle, but using more suitable methods for prescribing intensity (such as VO2max and HRres) seems to mitigate the effects of the lack of progression in training.

Finally, our study exposes some limitations that need to be highlighted. Although the general analysis has a considerable number of studies, some subanalyses were carried out with a small number of studies. When assessing methodological quality, the set of studies analyzed did not clearly report most of the information, and of the five evaluation criteria, three were reported unclearly in more than $50 \%$ of the studies, making it difficult to assess the risk of bias. Another limitation is related to the lack of important information in the studies, which prevent association with the results, such as disease duration, antihypertensive drugs used and the training status of the participants.

On the other hand, the present study has some strengths. As far as we know, this is a first meta-analysis that assesses the effects of the progression of aerobic training in patients with hypertension, with a significant number of participants being analyzed. The analysis of possible moderators of the effect using the meta-regression analysis is also a strong point of the study. In practical terms, although some guidelines recommend the progression of training, more precise information is lacking in relation to the way to progress. In this regard, the present study presents results that will possibly assist in the prescription of exercises, such as manipulation and the increase of the variables of the training, especially session length and intensity, thus maximizing the beneficial effects of exercise on BP.

\section{Conclusion}

Aerobic training promotes a reduction in SBP and DBP levels in adults with hypertension, regardless of whether there is progression of the training variables. However, when manipulating the training variables, a response of greater magnitude seems to occur with the progression in duration, for SBP, and in intensity, for DBP. Nevertheless, although there is no chronic difference, the progression of the training variables must be considered, in order to potentiate the effects caused by aerobic training on the pressure response.

\section{Declarations}

\section{Availability of data and material}

Please contact the authors for data requests.

\section{Acknowledgements}

Not applicable.

\section{Author's contribution}

GTB - Participated in the literary search, data extraction, in date analysis, interpretation of dates for the work and writing of the manuscript.

$\mathrm{IH}$ - Participated in the literary search, data extraction, in date analysis, interpretation of dates for the work and writing of the manuscript.

JCC - Participated in the literary search, data extraction, interpretation of dates for the work and writing of the manuscript.

BAV - Participated in the literary search, data extraction, interpretation of dates for the work and writing of the manuscript. 
RSD - Participated in the initial study design, interpretation of dates for the work and critical review of the manuscript.

AMG - Participated in the initial study design, interpretation of dates for the work and critical review of the manuscript.

All authors reviewed final version of the manuscript, takes responsibility for the integrity of the data and the accuracy of the data analysis. All authors read and approved the final manuscript.

\section{Funding}

No sources of funding were used to assist in the preparation of this article.

\section{Conflicts of interest}

No, I declare that the authors have no competing interests as defined by Nature Research, or other interests that might be perceived to influence the results and/or discussion reported in this paper.

\section{References}

1. Mills, K. T. et al. Global Disparities of Hypertension Prevalence and Control: A Systematic Analysis of Population-Based Studies From 90 Countries. Circulation 134, 441-450 (2016).

2. Rapsomaniki, E. et al. Blood pressure and incidence of twelve cardiovascular diseases: lifetime risks, healthy life-years lost, and age-specific associations in 1·25 million people. The Lancet 383, 1899-1911 (2014).

3. Pan, H., Hibino, M., Kobeissi, E. \& Aune, D. Blood pressure, hypertension and the risk of sudden cardiac death: a systematic review and meta-analysis of cohort studies. Eur J Epidemio/ 35, 443-454 (2020).

4. Whelton, P. K. et al. 2017 ACC/AHA/AAPA/ABC/ACPM/AGS/APhA/ASH/ASPC/NMA/PCNA Guideline for the Prevention, Detection, Evaluation, and Management of High Blood Pressure in Adults. Hypertension 71, e127-e248 (2018).

5. Brook, R. D. et al. Beyond Medications and Diet: Alternative Approaches to Lowering Blood Pressure: A Scientific Statement From the American Heart Association. Hypertension 61, 1360-1383 (2013).

6. Barroso, W., Rodrigues, C. \& Bortolotto, L. Diretrizes Brasileiras de Hipertensão Arterial - 2020. Arq Bras Cardiol. 138 (2020).

7. MacDonald, H. V. \& Pescatello, L. S. Exercise Prescription for Hypertension: New Advances for Optimizing Blood Pressure Benefits. in Lifestyle in Heart Health and Disease Academic. Academic Press, 115-136. doi:10.1016/B978-0-12-8112793.00009-4 (2018).

8. Williams, B. et al. 2018 ESC/ESH Guidelines for the management of arterial hypertensionThe Task Force for the management of arterial hypertension of the European Society of Cardiology (ESC) and the European Society of Hypertension (ESH). Eur Heart J 39, 3021-3104 (2018).

9. Rabi, D. M. et al. Hypertension Canada's 2020 Comprehensive Guidelines for the Prevention, Diagnosis, Risk Assessment, and Treatment of Hypertension in Adults and Children. Can J Cardio/36, 596-624 (2020).

10. Unger, T. et al. 2020 International Society of Hypertension Global Hypertension Practice Guidelines. Hypertension 75, 3341357 (2020).

11. Cornelissen, V. A. \& Smart, N. A. Exercise training for blood pressure: a systematic review and meta-analysis. Am Heart J 2, e004473 (2013).

12. Igarashi, Y., Akazawa, N. \& Maeda, S. Regular aerobic exercise and blood pressure in East Asians: A meta-analysis of randomized controlled trials. Clin Exp Hypertens 40, 378-389 (2018).

13. Cao, L. et al. The effectiveness of aerobic exercise for hypertensive population: A systematic review and meta-analysis. $J$ Clin Hypertens 21, 868-876 (2019).

14. Sharman, J. E., Smart, N. A., Coombes, J. S. \& Stowasser, M. Exercise and sport science australia position stand update on exercise and hypertension. J Hum Hypertens 33, 837-843 (2019). 
15. Bahmanbeglou, N., Ebrahim, K., Maleki, M., Nikpajouh, A. \& Ahmadizad, S. Short-Duration High-Intensity Interval Exercise Training Is More Effective Than Long Duration for Blood Pressure and Arterial Stiffness But Not for Inflammatory Markers and Lipid Profiles in Patients With Stage 1 Hypertension: J Cardiopulm Rehabil Prev 39, 50-55 (2019).

16. Gorostegi-Anduaga, I. et al. Effects of different aerobic exercise programmes with nutritional intervention in sedentary adults with overweight/obesity and hypertension: EXERDIET-HTA study. Eur J Prev Cardiol 25, 343-353 (2018).

17. Costa, E. C. et al. Effects of High-Intensity Interval Training Versus Moderate-Intensity Continuous Training On Blood Pressure in Adults with Pre- to Established Hypertension: A Systematic Review and Meta-Analysis of Randomized Trials. Sports Med 48, 2127-2142 (2018).

18. Börjesson, M., Onerup, A., Lundqvist, S. \& Dahlöf, B. Physical activity and exercise lower blood pressure in individuals with hypertension: narrative review of 27 RCTs. Br J Sports Med 50, 356-361 (2016).

19. Boutcher, Y. N. \& Boutcher, S. H. Exercise intensity and hypertension: what's new? J Hum Hypertens 31, 157-164 (2017).

20. Garber, C. E. et al. Quantity and Quality of Exercise for Developing and Maintaining Cardiorespiratory, Musculoskeletal, and Neuromotor Fitness in Apparently Healthy Adults: Guidance for Prescribing Exercise. Med Sci Sports Exerc 43, 13341359 (2011).

21. Moher, D., Liberati, A., Tetzlaff, J. \& Altman, D. G. Preferred Reporting Items for Systematic Reviews and Meta-Analyses: The PRISMA Statement. Ann Int Med. 151, 264-9 (2009).

22. Higgins, J. P. \& Green, S. Cochrane Handbook for Systematic Reviews of Interventions: Cochrane Book Series. 674.

23. Abdelaal, A. A. M. \& Mohamad, M. A. Obesity indices and haemodynamic response to exercise in obese diabetic hypertensive patients: Randomized controlled trial. Obes Res Clin Pract 9, 475-486 (2015).

24. Baghaiee, B. et al. Effects of a 12-week aerobic exercise on markers of hypertension in men. J Cardiovasc Thorac Res 10, 162-168 (2018).

25. Farahani, A. V. et al. The Effects of a 10-Week Water Aerobic Exercise on the Resting Blood Pressure in Patients with Essential Hypertension. Asian J Sports Med 1, (2010).

26. Hagberg, J. M., Montain, S. J., Martin, W. H. \& Ehsani, A. A. Effect of exercise training in 60- to 69-year-old persons with essential hypertension. Am J Cardio/ 64, 348-353 (1989).

27. Kokkinos, P. F. et al. Effects of Regular Exercise on Blood Pressure and Left Ventricular Hypertrophy in African-American Men with Severe Hypertension. N Engl J Med 333, 1462-1467 (1995).

28. Lamina, S. Effects of Continuous and Interval Training Programs in the Management of Hypertension: A Randomized Controlled Trial. J Clin Hypertens 12, 841-849 (2010).

29. Latosik, E. et al. Physiological Responses Associated with Nordic-Walking Training in Systolic Hypertensive Postmenopausal Women. J Hum Kinet 43, 185-190 (2014).

30. De Meirelles, L. R. et al. Chronic exercise reduces platelet activation in hypertension: upregulation of the l-arginine-nitric oxide pathway: Exercise in hypertension. Scand J Med Sci Sports 19, 67-74 (2008).

31. Soltani, M., Aghaei Bahmanbeglou, N. \& Ahmadizad, S. High-intensity interval training irrespective of its intensity improves markers of blood fluidity in hypertensive patients. Clin Exp Hypertens 42, 309-314 (2020).

32. Tanaka, H. et al. Swimming training lowers the resting blood pressure in individuals with hypertension. $\mathbf{J}$ Hypertens $\mathbf{1 5}$, 651-657 (1997).

33. Turner, M. J., Spina, R. J., Kohrt, W. M. \& Ehsani, A. A. Effect of Endurance Exercise Training on Left Ventricular Size and Remodeling in Older Adults With Hypertension. J Gerontol A Biol Sci Med Sci 55, M245-M251 (2000).

34. Wong, A. et al. The effects of swimming training on arterial function, muscular strength, and cardiorespiratory capacity in postmenopausal women with stage 2 hypertension: Menopause 26, 653-658 (2019).

35. Arca, E. A., Martinelli, B., Martin, L. C., Waisberg, C. B. \& Franco, R. J. da S. Aquatic Exercise is as Effective as dry Land Training to Blood Pressure Reduction in Postmenopausal Hypertensive Women: Aquatic Exercise and Hypertension. Physiother Res Int 19, 93-98 (2014). 
36. He, L., Wei, W. ren \& Can, Z. Effects of 12-week brisk walking training on exercise blood pressure in elderly patients with essential hypertension: a pilot study. Clin Exp Hypertens 40, 673-679 (2018).

37. Hong, S., Lee, D. \& Lee, G. Effect of Aerobic Exercise on Blood Pressure and Arterial Compliance in Patients with Essential Hypertension. J Exerc Physio/ 21, 9-18 (2018).

38. Khalid, T. Effects of Exercise Training on Postmenopausal Hypertension: Implications on Nitric Oxide Levels. Med J Malaysia 68, 459-464 (2013).

39. Izadi, M. R., Ghardashi Afousi, A., Asvadi Fard, M. \& Babaee Bigi, M. A. High-intensity interval training lowers blood pressure and improves apelin and NOx plasma levels in older treated hypertensive individuals. J Physiol Biochem 74, 47-55 (2018).

40. Koga, M. et al. Mild exercise decreases plasma endogenous digitalislike substance in hypertensive individuals. Hypertension 19, II231-II231 (1992).

41. Maruf, F. A., Akinpelu, A. O. \& Salako, B. L. A Randomized Controlled Trial of the Effects of Aerobic Dance Training on Blood Lipids Among Individuals with Hypertension on a Thiazide. High Blood Press Cardiovasc Prev 21, 275-283 (2014).

42. Miura, S. et al. Urinary kallikrein activity is increased during the first few weeks of exercise training in essential hypertension. J Hypertens 12, 815-823 (1994).

43. Ramos, R. M. et al. Moderate Aerobic Training Decreases Blood Pressure but No Other Cardiovascular Risk Factors in Hypertensive Overweight/Obese Elderly Patients. Gerontol Geriatr Med 4, 233372141880864 (2018).

44. Tanabe, Y. et al. Changes in Serum Concentrations of Taurine and Other Amino Acids in Clinical Antihypertensive Exercise Therapy. Clin Exp Hypertens 11, 149-165 (1989).

45. Tsai, J.-C. et al. Beneficial effect on blood pressure and lipid profile by programmed exercise training in taiwanese patients with mild hypertension. Clin Exp Hypertens 24, 315-324 (2002).

46. Tsai, J. et al. The Beneficial Effect of Regular Endurance Exercise Training on Blood Pressure and Quality of Life in Patients with Hypertension. Clin Exp Hypertens 26, 255-265 (2004).

47. Naci, H. et al. How does exercise treatment compare with antihypertensive medications? A network meta-analysis of 391 randomised controlled trials assessing exercise and medication effects on systolic blood pressure. Br J Sports Med 53, 859-869 (2019).

48. Ettehad, D. et al. Blood pressure lowering for prevention of cardiovascular disease and death: a systematic review and meta-analysis. The Lancet 387, 957-967 (2016).

49. Hellsten, Y. \& Nyberg, M. Cardiovascular Adaptations to Exercise Training. Compr Physio/ 6, 1-31. doi:10.1002/cphy.c140080 (2016).

50. Pescatello, L. S., MacDonald, H. V., Lamberti, L. \& Johnson, B. T. Exercise for Hypertension: A Prescription Update Integrating Existing Recommendations with Emerging Research. Curr Hypertens Rep 17, 1-10 (2015).

51. Eicher, J. D., Maresh, C. M., Tsongalis, G. J., Thompson, P. D. \& Pescatello, L. S. The additive blood pressure lowering effects of exercise intensity on post-exercise hypotension. Hypertension 160, 513-520 (2010).

52. Ishikawa-Takata, K. How much exercise is required to reduce blood pressure in essential hypertensives: a dose-response study. Am J Hypertens 16, 629-633 (2003).

53. Pescatello, L. S. et al. Physical Activity to Prevent and Treat Hypertension: A Systematic Review. Am J Hypertens 51, 1314-1323 (2019).

54. Turnbull, F. et al. Do men and women respond differently to blood pressure-lowering treatment? Results of prospectively designed overviews of randomized trials. Eur Heart J 29, 2669-2680 (2008).

55. Wang, J. et al. Prevalence and Risk Factors of Comorbidities among Hypertensive Patients in China. Int J Med Sci 14, 201-212 (2017). 
56. Moraes-Silva, I. C., Mostarda, C. T., Silva-Filho, A. C. \& Irigoyen, M. C. Hypertension and Exercise Training: Evidence from Clinical Studies. Adv Exp Med Biol, 65-84 (2017).

57. Sardeli, A. V. et al. Do baseline blood pressure and type of exercise influence level of reduction induced by training in hypertensive older adults? A meta-analysis of controlled trials. Exp Gerontol 140, 111052 (2020).

58. Leal, J. M., Galliano, L. M. \& Del Vecchio, F. B. Effectiveness of High-Intensity Interval Training Versus Moderate-Intensity Continuous Training in Hypertensive Patients: a Systematic Review and Meta-Analysis. Curr Hypertens Rep 22, (2020).

59. Reichert, T. et al. Aquatic Training in Upright Position as an Alternative to Improve Blood Pressure in Adults and Elderly: A Systematic Review and Meta-Analysis. Sports Med 48, 1727-1737 (2018).

60. McConnell, K. J. 2017 ACC/AHA guidelines for BP management in adult patients. Pharmacy Today 24, 57-73 (2018).

61. Kelley, G. A. \& Kelley, K. S. Brief Report: Exercise and Blood Pressure in Older Adults-An Updated Look. Int J Hypertens 2018, 1-5 (2018).

62. Thomopoulos, C., Parati, G. \& Zanchetti, A. Effects of blood pressure-lowering treatment on cardiovascular outcomes and mortality: 13 - benefits and adverse events in older and younger patients with hypertension. J Hypertens 36, 1622-1636 (2018).

\section{Figures}

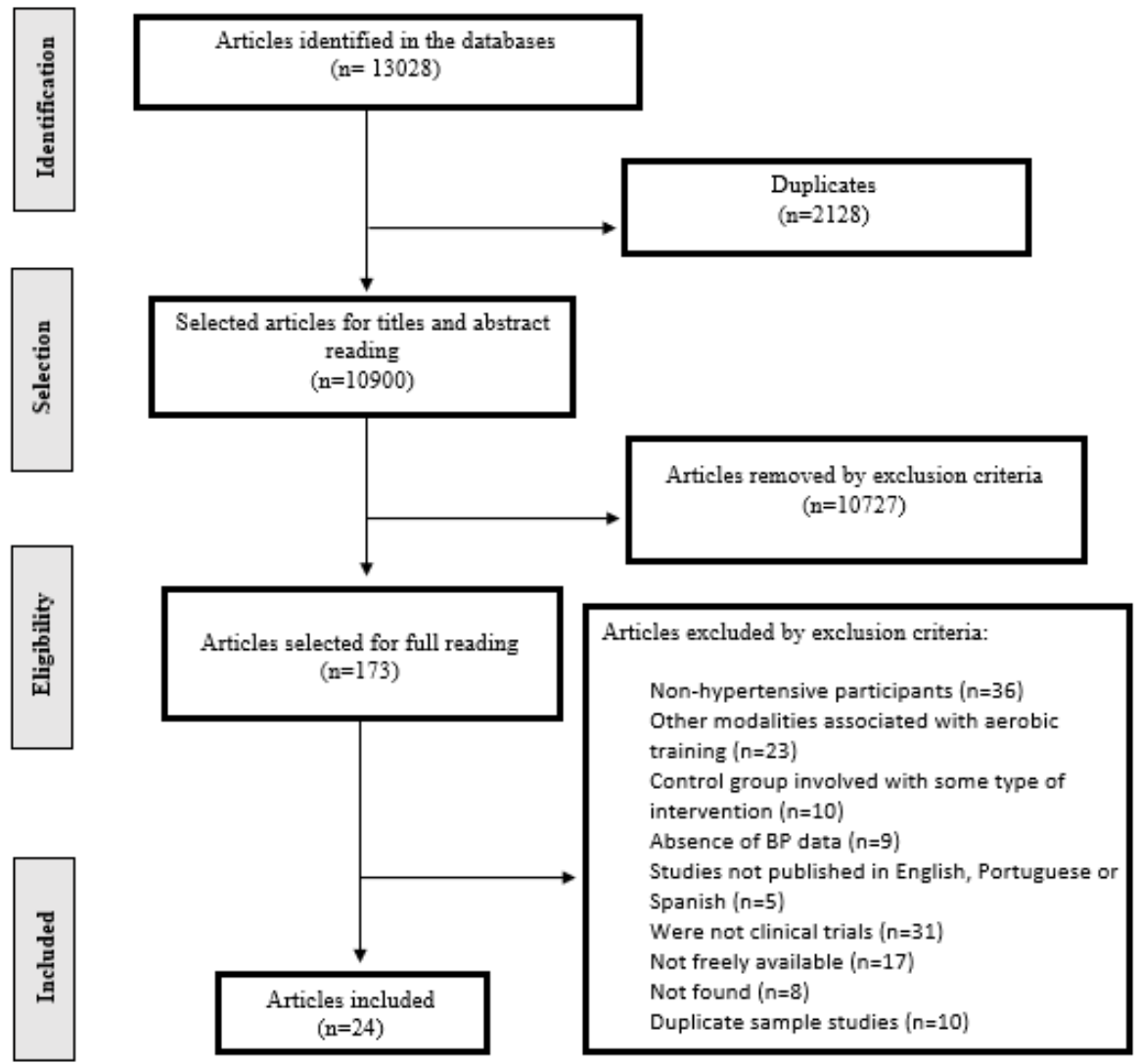

\section{Figure 1}

Flowchart with information on the different phases of the systematic review 
A

Studies

Abdelaal and Mohamad 2014

Baghaiee et al 2018

Farahani et al 2010

Hagberg et al 1989

Kokkinos et al a 1995

Kokkinos et al b 1995

Lamina et al a 2010

Lamina et al b 2010

Latosik et al 2014

Meirelles et al 2009

Soltani et al a 2019

Soltani et al b 2018

Tanaka et al 1997

Tumer et al 2000

Wong et al 2018

Overall $\left(\|^{\wedge} 2=99.12 \%, p<0.001\right)$

\section{B}

Studies

Abdelaal and Mohamad 2014

Baghaiee et al 2018

Farahani et al 2010

Hagberg et al 1989

Kokkinos et al a 1995

Kokkinos et al b 1995

Lamina et al a 2010

Lamina et al b 2010

Latosik et al 2014

Meirelles et al 2009

Soltani et al a 2019

Soltani et al b 2019

Tanaka et al 1997

Tumer et al 2000

Wong et al 2018

Overall $\left(\left.\right|^{\wedge} 2=98.57 \%, P<0.001\right)$
Estimate (95s C.I.)

$\begin{array}{lll}-7.260 \quad(-9.475, & -5.045)\end{array}$

$-0.600 \quad(-0.789,-0.411)$

$-14.890(-24.504,-5.276)$

$-5.000(-23.814,13.814)$

$-6.000(-16.691,4.691)$

$-5.000(-16.362,6.362)$

$-18.300(-24.436,-12.164)$

$-17.030(-23.461,-10.599)$

$-3.600(-13.172,5.972)$

$-26.300(-33.512,-19.088)$

$-10.000(-25.569, \quad 5.569)$

$-10.000(-25.899,5.899)$

$-5.000(-22.751,12.751)$

$-13.000(-23.418,-2.582)$

$-12.000(-12.555,-11.445)$

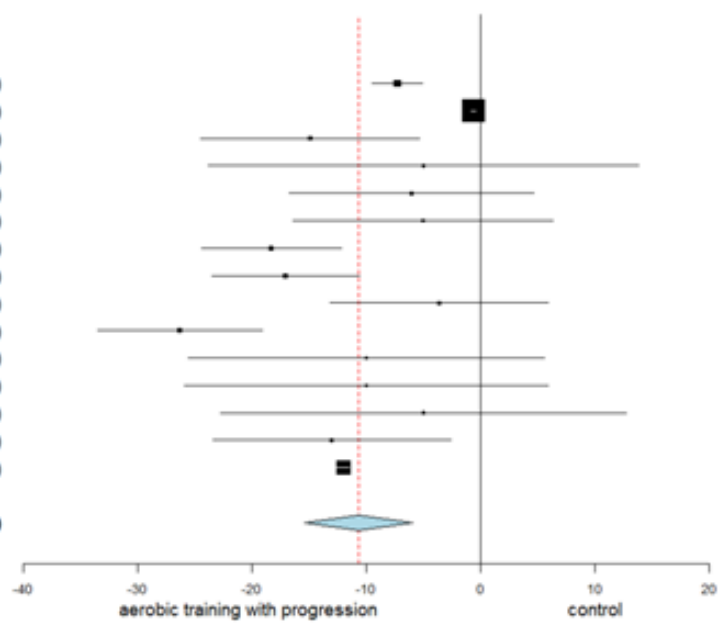

Estimate (95: C.I.)

$-5.860 \quad(-7.090,-4.630)$

$-0.029(-0.275,0.217)$

$-4.350(-11.087,2.387)$

$-8.000(-17.219,1.219)$

$-7.000(-13.190,-0.810)$

$-5.000(-11.439,1.439)$

$-0.750 \quad(-2.022,0.522)$

$-5.160 \quad(-7.416,-2.904)$

$-1.400 \quad(-7.500,4.700)$

$-13.400(-16.136,-10.664)$

$-2.940(-12.294,6.414)$

$-11.030(-22.668,0.608)$

$-2.000(-14.854,10.854)$

$-7.300(-15.505,0.905)$

$-9.000 \quad(-9.555,-8.445)$

$-5.487 \quad(-8.663,-2.310)$

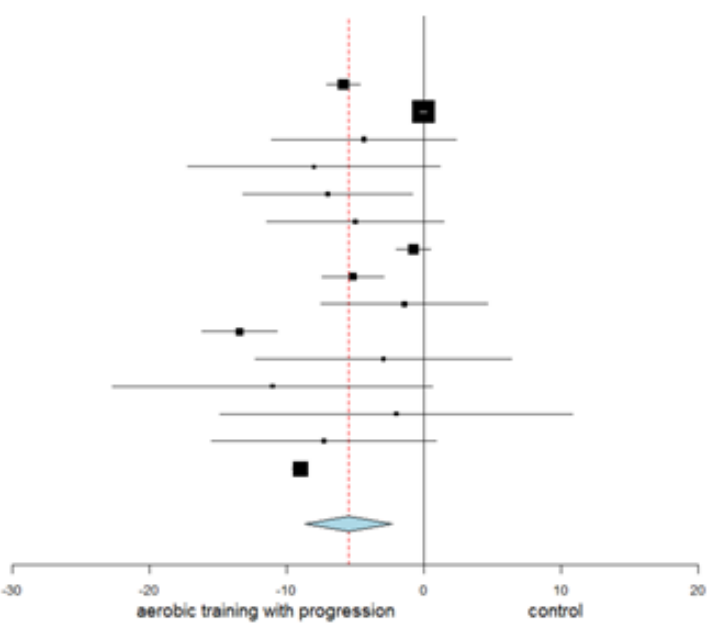

Figure 2

Mean differences in SBP (A) and DPB (B) observed between aerobic training with progression compared to control (without intervention). Study-specific estimates (black square); pooled estimates of random-effects meta-analyses (blue diamond).Cl indicates confidence interval 
A

Studies

Estimate (95\& C.I.)

Arca et al a 2014

Arca et al b 2014

He et al 2018

Hong et al 2018

Khalid et al 2013

Izadi et al 2017

Koga et al 1992

Maruf et al 2014

Miura et al 1994

Ramos et al 2018

Tanabe et al 1989

Tsai et al a 2002

Tsai et al b 2004

$-21.000(-42.429, \quad 0.429)$

$-21.000(-43.239,1.239)$

$-10.500(-14.755,-6.245)$

$-5.000(-25.108,15.108)$

$-15.000(-21.827,-8.173)$

$-4.100(-10.552,2.352)$

$-9.000(-26.748,8.748)$

$-9.960(-18.499,-1.421)$

$-6.000(-19.718,7.718)$

$-10.500(-14.046,-6.954)$

$-11.600(-26.739, \quad 3.539)$

$-10.000(-21.668,1.668)$

$-9.500(-16.968,-2.032)$

Overall $\left(\left.\right|^{\wedge} 2=0 \%, P=0.788\right)-10.167 \quad(-12.213,-8.120)$

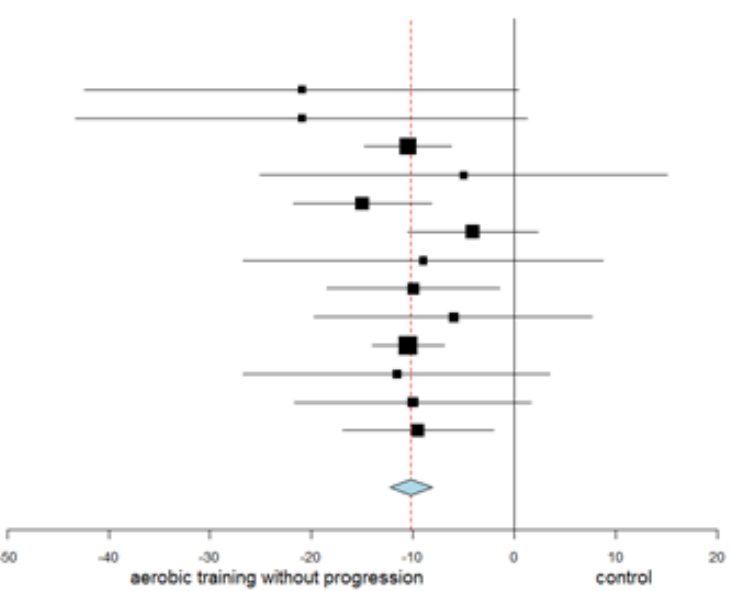

B

Studies

Arca et al a 2014

Arca et al b 2014

He et al 2018

Hong et al 2018

Khalid et al 2013

Izadi et al 2017

Koga et al 1992

Maruf et al 201

Miura et al 1994

Ramos et al 2018

Tanabe et al 1989

Tsai et al a 2002

Tsai et al b 2004

Overall $\left(1^{\wedge} 2=60.8 \%, P=0.002\right)$

Estimate (95t C.I.)

$-5.000(-19.500,9.500)$

$-3.000(-18.081,12.081)$

$-1.900 \quad(-5.137,1.337)$

$-2.710(-14.505, \quad 9.085)$

$-9.000(-13.896,-4.104)$

$-3.060 \quad(-6.990,0.870)$

$-8.000(-15.064,-0.936)$

$-3.380 \quad(-9.511,2.751)$

$-4.000(-13.995, \quad 5.995)$

$-11.400(-14.081,-8.719)$

$-5.400(-17.188,6.388)$

$-10.300(-19.404,-1.196)$

$-10.300(-13.992,-6.608)$

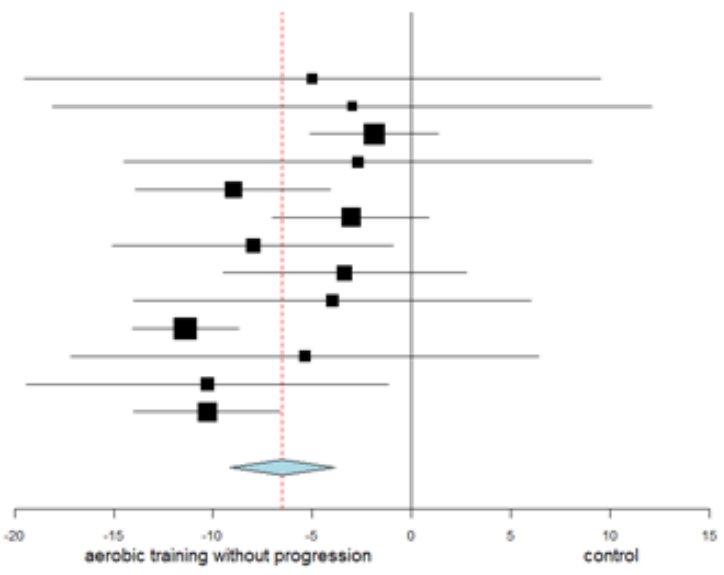

\section{Figure 3}

Mean differences in SBP (A) and DBP (B) observed between aerobic training without progression compared to control (without intervention). Study-specific estimates (black square); pooled estimates of random-effects meta-analyses (blue diamond).Cl indicates confidence interval

\section{Supplementary Files}

This is a list of supplementary files associated with this preprint. Click to download.

- Supplementaryinformation.docx 